\title{
Non-perturbative effective interactions from fluxes
}

\author{
Marco Billò ${ }^{1}$, Livia Ferro ${ }^{1,2}$, Marialuisa Frau ${ }^{1}$, Francesco Fucito ${ }^{3}$, \\ Alberto Lerda ${ }^{4}$ and Jose F. Morales ${ }^{3}$ \\ ${ }^{1}$ Dipartimento di Fisica Teorica, Università di Torino \\ and I.N.F.N. - sezione di Torino \\ Via P. Giuria 1, I-10125 Torino, Italy \\ ${ }^{2}$ Laboratoire de Physique Théorique \\ École Normale Supérieure \\ 24, rue Lhomond, F- 75231 Paris Cedex 05, France \\ ${ }^{3}$ I.N.F.N. - sezione di Roma II \\ Via della Ricerca Scientifica, I-00133 Roma, Italy \\ ${ }^{4}$ Dipartimento di Scienze e Tecnologie Avanzate, Università del Piemonte Orientale \\ and I.N.F.N. - Gruppo Collegato di Alessandria - sezione di Torino \\ Via V. Bellini 25/G, I-15100 Alessandria, Italy \\ billo, ferro, frau, lerda@to.infn.it, \\ Francesco.Fucito, Francisco.Morales@roma2.infn.it
}

\begin{abstract}
Motivated by possible implications on the problem of moduli stabilization and other phenomenological aspects, we study D-brane instanton effects in flux compactifications. We focus on a local model and compute non-perturbative interactions generated by gauge and stringy instantons in a $\mathcal{N}=1$ quiver theory with gauge group $\mathrm{U}\left(N_{0}\right) \times \mathrm{U}\left(N_{1}\right)$ and matter in the bifundamentals. This model is engineered with fractional D3-branes at a $\mathbb{C}^{3} /\left(\mathbb{Z}_{2} \times \mathbb{Z}_{2}\right)$ singularity and its non-perturbative sectors are described by introducing fractional D-instantons. We find a rich variety of instanton-generated interactions, ranging from superpotentials and Beasley-Witten like multi-fermion terms to non-supersymmetric flux-induced instanton interactions.
\end{abstract}

KeYwords: Superstrings, D-branes, Gauge Theories, Instantons. 


\section{Contents}

1. Introduction and motivations 1

2. D3/D(-1)-branes on $\mathbb{C}^{3} /\left(\mathbb{Z}_{2} \times \mathbb{Z}_{2}\right) \quad$ 5

2.1 The gauge theory 5

$\begin{array}{lll}2.2 & \text { The instanton moduli space } & 8\end{array}$

3. D-instanton partition function $\quad 11$

3.1 The moduli space integral 12

$\begin{array}{lll}3.2 & \text { Zero-mode counting for gauge instantons } & 15\end{array}$

4. Effective interactions from gauge instantons $\quad \mathbf{1 6}$

$\begin{array}{lll}4.1 \text { The gauge instanton action } & 16\end{array}$

4.2 Field theory results: non-perturbative F-terms 20

5. Non-perturbative flux-induced effective interactions 21

5.1 One-instanton effects with $G \neq 0 \quad 22$

5.2 One-instanton effects with $\bar{G} \neq 0 \quad 24$

6. Stringy instanton effects in presence of fluxes 25

A. Appendix $\quad \mathbf{2 7}$

$\begin{array}{ll}\text { A.1 Gauge coupling and instanton action } & 27\end{array}$

A.2 Vertex operators for gauge fields and instanton moduli 28

A.3 Derivation of the non-perturbative flux effects for $N_{f}=N_{c}-1 \quad 30$

A.4 Derivation of the non-perturbative flux effects for $N_{f}=N_{c} \quad 31$

\section{Introduction and motivations}

Recently a lot of attention has been devoted to the study of four dimensional compactifications of Type II string theories with systems of intersecting or magnetized D-branes that preserve $\mathcal{N}=1$ supersymmetry $[1,2,3]$. These compactifications provide, in fact, promising scenarios for phenomenological applications and realistic model building in which gauge interactions similar to those of the supersymmetric extensions of the Standard Model of particle physics are engineered using space-filling D-branes that partially or totally wrap the internal six-dimensional space. The effective actions of such brane-world models describe interactions of gauge degrees of freedom, associated to open strings, with gravitational fields, associated to closed strings, and have the generic structure of $\mathcal{N}=1$ supergravity 
in four dimensions coupled to vector and chiral multiplets. As is well-known [4], fourdimensional $\mathcal{N}=1$ supergravity theories are specified by the choice of a gauge group $\mathcal{G}$, with the corresponding adjoint fields and gauge kinetic functions, by a Kähler potential $K$ and a superpotential $W$, which are, respectively, a real and a holomorphic function of some chiral superfields $\Phi^{i}$. The supergravity vacuum is parametrized by the expectation values of these chiral multiplets that minimize the scalar potential

$$
V=\mathrm{e}^{K}\left(D_{i} \bar{W} D^{i} W-3|W|^{2}\right)+D^{a} D_{a}
$$

where $D_{i} W \equiv \partial_{\Phi^{i}} W+\left(\partial_{\Phi^{i}} K\right) W$ is the Kähler covariant derivative of the superpotential and the $D^{a}(a=1, \ldots, \operatorname{dim}(\mathcal{G}))$ are the D-terms. Supersymmetric vacua, in particular, correspond to those solutions of the equations $\partial_{\Phi^{i}} V=0$ satisfying the D- and F-flatness conditions $D^{a}=D_{i} W=0$.

When we consider Type IIB string theory on a Calabi-Yau three-fold in presence of D3-branes, which is the case discussed in this paper, the chiral superfields $\Phi^{i}$ comprise the fields $U^{r}$ and $T^{m}$ that parameterize the deformations of the complex and Kähler structures of the three-fold, the axion-dilaton field

$$
\tau=C_{0}+\mathrm{ie}^{-\varphi}
$$

where $C_{0}$ is the R-R scalar and $\varphi$ the dilaton, and also some multiplets $\Phi_{\text {open }}$ coming from the open strings attached to the D-branes. The resulting low-energy $\mathcal{N}=1$ supergravity model has a highly degenerate vacuum. One way to lift (at least partially) this degeneracy is provided by the addition of internal 3 -form fluxes of the bulk theory $[5,6,7]$ via the generation of a superpotential $[8,9]$

$$
W_{\text {flux }}=\int G_{3} \wedge \Omega .
$$

Here $\Omega$ is the holomorphic (3,0)-form of the Calabi-Yau three-fold and $G_{3}=F-\tau H$ is the complex 3-form flux given in terms of the R-R and NS-NS fluxes $F$ and $H$. The flux superpotential (1.3) depends explicitly on $\tau$ and implicitly on the complex structure parameters $U^{r}$ which specify $\Omega$. Insisting on unbroken $\mathcal{N}=1$ supersymmetry requires the flux $G_{3}$ to be an imaginary anti-selfdual 3-form of type $(2,1)$ [10], since the F-terms $D_{U^{r}} W_{\text {flux }}, D_{\tau} W_{\text {flux }}$ and $D_{T^{m}} W_{\text {flux }}$ are proportional to the $(1,2),(3,0)$ and $(0,3)$ components of the $G$-flux respectively. These F-terms can also be interpreted as the "auxiliary" $\theta^{2}$-components of the kinetic functions for the gauge theory defined on the space-filling branes, and thus are soft supersymmetry breaking terms for the brane-world effective action. Such soft terms have been computed in various scenarios of flux compactifications and their effects, like for instance induced masses for the gauginos and the gravitino, have been analyzed relying on the structure of the bulk supergravity Lagrangian and on $\kappa$-symmetry considerations (see for instance the reviews $[5,6,7]$ and references therein) and recently also by a direct world-sheet analysis in [11].

Beside fluxes, also non-perturbative contributions $[12,13]$ to the effective actions may play an important rôle in the moduli stabilization process $[14,15]$ and bear phenomenologically relevant implications for string theory compactifications. In the framework we 
are considering, non-perturbative sectors are described by configurations of D-instantons or, more generally, by wrapped Euclidean branes which may lead to the generation of a non-perturbative superpotential of the form

$$
W_{\text {n.p. }}=\sum_{\left\{k_{A}\right\}} c_{\left\{k_{A}\right\}}\left(\Phi^{i}\right) \mathrm{e}^{2 \pi \mathrm{i} \sum_{A} k_{A} \tau_{A}} .
$$

Here the index $A$ labels the cycles wrapped by the instantonic branes, while $k_{A}$ denotes the instanton number and $\tau_{A}$ the complexified gauge coupling of a D-brane wrapping the cycle $A$. Finally, $c_{\left\{k_{A}\right\}}\left(\Phi^{i}\right)$ are some (holomorphic) functions of the chiral superfields whose particular form depends on the details of the model. In general, the $\tau_{A}$ 's depend on the axion-dilaton modulus $\tau$ and the Kähler parameters $T^{m}$ that describe the volumes of the cycles around which the D-branes are wrapped ${ }^{1}$. We remark that Eq. (1.4) holds both for gauge and stringy instantons corresponding, respectively, to the cases where the cycle $A$ is occupied or not by a gauge D-brane.

The interplay of fluxes and non-perturbative contributions, leading to a combined superpotential

$$
W=W_{\text {flux }}+W_{\text {n.p. }},
$$

offers new possibilities for finding supersymmetric vacua. Indeed, the derivatives $D_{U^{r}} W_{\text {flux }}$, $D_{\tau} W_{\text {flux }}$ and $D_{T^{m}} W_{\text {flux }}$ might now be compensated by $D_{U^{r}} W_{\text {n.p. }}, D_{\tau} W_{\text {n.p. }}$ and $D_{T^{m}} W_{\text {n.p. }}$. $[15]$ so that also the $(1,2),(3,0)$ and $(0,3)$ components of $G_{3}$ may become compatible with supersymmetry and help in removing the vacuum degeneracy [16].

Another option could be to arrange things in such a way to have a Minkowski vacuum with $V=0$ and broken supersymmetry. If the superpotential is divided into an observable and a hidden sector, with the flux-induced supersymmetry breaking happening in the latter, this could be a viable model for supersymmetry breaking mediation. If all moduli are present in $W$, the number of equations necessary to satisfy the extremality condition for $V$ seems sufficient to obtain a complete moduli stabilization. To fully explore these, or other, possibilities, it is crucial however to develop reliable techniques to compute nonperturbative corrections to the effective action and determine the detailed structure of the non-perturbative superpotentials that can be generated, also in presence of background fluxes.

In the last few years there has been much progres in the analysis of non-perturbative effects in brane-world models and concrete computational tools have been developed using systems of branes with different boundary conditions $[17,18]$. These methods not only allow to reproduce [18]-[22] the known instanton calculus of (supersymmetric) field theories [23], but can also be generalized to more exotic configurations where field theory methods are not yet available [24]- [46]. The study of these exotic instantons has led to interesting results in relation to moduli stabilization, (partial) supersymmetry breaking and even fermion masses and Yukawa couplings [24, 25, 33]. A careful analysis of the moduli of this kind of instantons is however required in order to be sure that unwanted neutral fermionic zeromodes are either absent, as in some orientifold models [28, 29, 30], or lifted [35, 39]. If

\footnotetext{
${ }^{1}$ The explicit dependence of $\tau_{A}$ on $\tau$ and $T^{m}$ can be derived from the Dirac-Born-Infeld action as explained in Appendix A.1.
} 
really generated, such exotic interactions could also become part of a scheme in which the supersymmetry breaking is mediated by non-perturbative soft-terms arising in the hidden sector of the theory, as recently advocated also in [46]. Nonetheless, the stringent conditions required for the non-perturbative terms to be different from zero, severely limit the freedom to engineer models which are phenomenologically viable.

To make this program more realistic, in this paper we address the study of the generation of non-perturbative terms in presence of fluxes. Indeed fluxes not only lead to the perturbative superpotential (1.3) but also lift some zero-modes of the instanton background and allow for new types of non-perturbative couplings. In the following we will consider the interactions generated by gauge and stringy instantons in a specific setup consisting of fractional D3-branes at a $\mathbb{C}^{3} /\left(\mathbb{Z}_{2} \times \mathbb{Z}_{2}\right)$ singularity which engineer a $\mathcal{N}=1 \mathrm{U}\left(N_{0}\right) \times \mathrm{U}\left(N_{1}\right)$ quiver gauge theory with bi-fundamental matter fields. In order to simplify the treatment, still keeping the desired supergravity interpretation, this quiver theory can thought of as a local description of a Type IIB Calabi-Yau compactification on the toroidal orbifold $T^{6} /\left(\mathbb{Z}_{2} \times \mathbb{Z}_{2}\right)$. From this local standpoint, it is not necessary to consider global restrictions on the number $N_{0}$ and $N_{1}$ of D3-branes, which can therefore be arbitrary, nor add orientifold planes for tadpole cancelation. In such a setup we then introduce background fluxes of type $G_{(3,0)}$ and $G_{(0,3)}$, and study the induced non-perturbative interactions in the presence of gauge and stringy instantons which we realize by means of fractional D-instantons. In this way we are able to obtain a very rich class of non-perturbative effects which range from "exotic" superpotentials terms to non-supersymmetric multi-fermion couplings. We also show that, as anticipated in [11], stringy instantons in presence of $G$-fluxes can generate non-perturbative interactions even for $\mathrm{U}(N)$ gauge theories. This has to be compared with the case without fluxes where an orientifold projection $[28,29,30]$ is required in order to solve the problem of the neutral fermionic zero-modes. Notice also that since the $G_{(3,0)}$ and $G_{(0,3)}$ components of the $G_{3}$ are related to the gaugino and gravitino masses (see for instance $[47,48]$ ), the non-perturbative flux-induced interactions can be regarded as the analog of the Affleck-Dine-Seiberg (ADS) superpotentials [49] for gauge/gravity theories with soft supersymmetry breaking terms. In particular the presence of a $G_{(0,3)}$ flux has no effect on the gauge theory at a perturbative level but it generates new instanton-mediated effective interactions [38].

For the sake of simplicity most of our computations will be carried out for instantons with winding number $k=1$; however we also briefly discuss some multi-instanton effects. In particular from a simple counting of zero-modes we find that in our quiver gauge theory an infinite tower of D-instanton corrections can contribute to the low-energy superpotential, even in the field theory limit with no fluxes, in constrast to what happens in theories with simple gauge groups where the ADS-like superpotentials are generated only by instantons with winding number $k=1$. These multi-instanton effects in the quiver theories certainly deserve further analysis and investigations.

The plan of the paper is the following: in Section 2 we review the D-brane setup in the orbifold $\mathbb{C}^{3} /\left(\mathbb{Z}_{2} \times \mathbb{Z}_{2}\right)$ in which our computations are carried out. In Section 3 we discuss a quick method to infer the structure of the non-perturbative contributions to the effective action based on dimensional analysis and symmetry considerations. In Section 4 we analyze 
the ADHM instanton action and discuss in detail the one-instanton induced interactions in SQCD-like models without introducing $G$-fluxes. Finally in Sections 5 and 6 we consider gauge and stringy instantons in presence of $G$-fluxes and compute the non-perturbative interactions they produce. Some more technical details are contained in the Appendix.

\section{D3/D(-1)-branes on $\mathbb{C}^{3} /\left(\mathbb{Z}_{2} \times \mathbb{Z}_{2}\right)$}

In this section we discuss the dynamics of the $\mathrm{D} 3 / \mathrm{D}(-1)$ brane system on the orbifold $\mathbb{C}^{3} /\left(\mathbb{Z}_{2} \times \mathbb{Z}_{2}\right)$ where the elements of $\mathbb{Z}_{2} \times \mathbb{Z}_{2}$ act on the three complex coordinates $z^{I}$ of $\mathbb{C}^{3}$ as follows

$$
\begin{aligned}
& h_{1}:\left(z^{1}, z^{2}, z^{3}\right) \rightarrow\left(z^{1},-z^{2},-z^{3}\right), \\
& h_{2}:\left(z^{1}, z^{2}, z^{3}\right) \rightarrow\left(-z^{1}, z^{2},-z^{3}\right), \\
& h_{3}:\left(z^{1}, z^{2}, z^{3}\right) \rightarrow\left(-z^{1},-z^{2}, z^{3}\right) .
\end{aligned}
$$

This material is well-known; nevertheless, we review it mainly with the purpose of setting our notations.

\subsection{The gauge theory}

A stack of $N$ D3-branes in flat space gives rise to a four-dimensional $\mathrm{U}(N)$ gauge theory with $\mathcal{N}=4$ supersymmetry. Its field content, corresponding to the massless excitations of the open strings attached to the D3-branes, can be organized into a $\mathcal{N}=1$ vector multiplet $V$ and three $\mathcal{N}=1$ chiral multiplets $\Phi^{I}(I=1,2,3)$. These are $N \times N$ matrices:

$$
\left\{V, \Phi^{I}\right\}_{v}^{u}
$$

with $u, v, \ldots=1, \ldots, N$. In $\mathcal{N}=1$ superspace notation, the action of the $\mathcal{N}=4$ theory is

$$
S=\frac{1}{4 \pi} \operatorname{Im}\left[\tau \int d^{4} x d^{2} \theta d^{2} \bar{\theta} \operatorname{Tr}\left(\bar{\Phi}_{I} \mathrm{e}^{2 V} \Phi^{I}\right)+\tau \int d^{4} x d^{2} \theta \operatorname{Tr}\left(\frac{1}{2} W^{\alpha} W_{\alpha}+\frac{1}{3 !} \epsilon_{I J K} \Phi^{I} \Phi^{J} \Phi^{K}\right)\right]
$$

where $\tau$ is the axion-dilaton field (1.2) and $W_{\alpha}=-\frac{1}{4} \bar{D}_{\dot{\alpha}} \bar{D}^{\dot{\alpha}} D_{\alpha} V$ is the chiral superfield whose lowest component is the gaugino.

When the D3-branes are placed in the $\mathbb{C}^{3} /\left(\mathbb{Z}_{2} \times \mathbb{Z}_{2}\right)$ orbifold, the supersymmetry of the gauge theory is reduced to $\mathcal{N}=1$ and only the $\left(\mathbb{Z}_{2} \times \mathbb{Z}_{2}\right)$-invariant components of $V$ and $\Phi^{I}$ are retained. Since $V$ is a scalar under the internal $\mathrm{SO}(6)$ group, while the chiral multiplets $\Phi^{I}$ form a vector, it is immediate to find the transformation properties of these fields under the orbifold group elements $h_{I}$. These are collected in Tab. 1, where in the first columns we have displayed the eigenvalues of $h_{I}$ and in the last column we have indicated the $\mathbb{Z}_{2} \times \mathbb{Z}_{2}$ irreducible representation $R_{A}(A=0,1,2,3)$ under which each field transforms ${ }^{2}$.

\footnotetext{
${ }^{2}$ Quantities carrying an index $A$ of the chiral or anti-chiral spinor representation of $\mathrm{SO}(6)$, like for example the gauginos $\Lambda^{\alpha A}$ or $\bar{\Lambda}_{\dot{\alpha} A}$ of the $\mathcal{N}=4$ theory, transform in the representation $R_{A}$ of the orbifold group; thus there is a one-to-one correspondence between the spinor indices of $\mathrm{SO}(6)$ and those labeling the irreducible representations of $\mathbb{Z}_{2} \times \mathbb{Z}_{2}$; for this reason we can use the same letters $A, B, \ldots$ in the two cases (see for example Ref. [11] for details).
} 


\begin{tabular}{cccccc}
\hline \hline fields & $h_{0}$ & $h_{1}$ & $h_{2}$ & $h_{3}$ & Rep's \\
\hline $\mathrm{V}$ & + & + & + & + & $R_{0}$ \\
$\Phi^{1}$ & + & + & - & - & $R_{1}$ \\
$\Phi^{2}$ & + & - & + & - & $R_{2}$ \\
$\Phi^{3}$ & + & - & - & + & $R_{3}$ \\
\hline \hline
\end{tabular}

Table 1: $\mathbb{Z}_{2} \times \mathbb{Z}_{2}$ eigenvalues of D3/D3 fields

To each representation $R_{A}$ of $\mathbb{Z}_{2} \times \mathbb{Z}_{2}$ one associates a fractional D3 brane type. Let $N_{A}$ be the number of D3-branes of type $A$ with

$$
\sum_{A=0}^{3} N_{A}=N
$$

so that the $N \times N$ adjoint fields $V, \Phi^{I}$ of the parent theory break into $N_{A} \times N_{B}$ blocks transforming in the representation $R_{A} \otimes R_{B}$. Explicitly, writing $A=(0, I)$ with $I=1,2,3$, we have

$$
R_{0} \otimes R_{A}=R_{A} \quad \text { and } \quad R_{I} \otimes R_{J}=\delta_{I J} R_{0}+\left|\epsilon_{I J K}\right| R_{K} .
$$

The invariant components of $V$ and $\Phi^{I}$ surviving the orbifold projection are given by those blocks where the non-trivial transformation properties of the fields are compensated by those of their Chan-Paton indices and are

$$
\{V\}_{v_{A}}^{u_{A}} \cup\left\{\Phi^{I}\right\}_{v_{A \otimes I}}^{u_{A}}
$$

Here the symbol \{\}$_{v_{B}}^{u_{A}}$ denotes the components of the $N_{A} \times N_{B}$ block, and the subindex $A \otimes I$ is a shorthand for the representation product $R_{A} \otimes R_{I}$, namely

$$
0 \otimes I=I \quad \text { and } \quad J \otimes I=\left|\epsilon_{J I K}\right| K
$$

as follows from (2.5). Eq. (2.6) represents the field content of a $\mathcal{N}=1$ gauge theory with gauge group $\prod_{A} \mathrm{U}\left(N_{A}\right)$ and matter in the bifundamentals $\left(N_{A}, \bar{N}_{B}\right)$, which is encoded in the quiver diagram displayed in Fig. 1.

The projected theory is invariant under the $\mathrm{U}(1)^{3}$ global symmetries corresponding to the Cartan subgroup of the $\mathrm{SO}(6) \mathcal{R}$-symmetry invariance of the $\mathcal{N}=4$ action (2.3):

$$
\begin{aligned}
& \Phi^{I} \rightarrow \mathrm{e}^{\mathrm{i} \zeta_{I}} \Phi^{I} \quad, \quad V \rightarrow V \quad, \quad W_{\alpha} \rightarrow \mathrm{e}^{\frac{\mathrm{i}}{2} \sum_{I} \zeta_{I}} W_{\alpha} \\
& d \theta \rightarrow \mathrm{e}^{-\frac{\mathrm{i}}{2} \sum_{I} \zeta_{I}} d \theta \quad, \quad d \bar{\theta} \rightarrow \mathrm{e}^{\frac{\mathrm{i}}{2} \sum_{I} \zeta_{I}} d \bar{\theta} ;
\end{aligned}
$$

these transformations encode the charges $q_{I}$ of the various fields w.r.t. to the three U(1)'s. The symmetry extends, as we will see, to the zero modes of the gauge fields in instantonic sectors, and can be exploited to constrain the form of the allowed non-perturbative interactions. To this aim, it will prove useful to take linear combinations of the $\mathrm{U}(1)^{3}$ symmetries (2.8) corresponding to introducing the charges

$$
q=q_{1}+q_{2}+q_{3}, \quad q^{\prime}=q_{1}-q_{2}, \quad q^{\prime \prime}=q_{1}-q_{3} .
$$




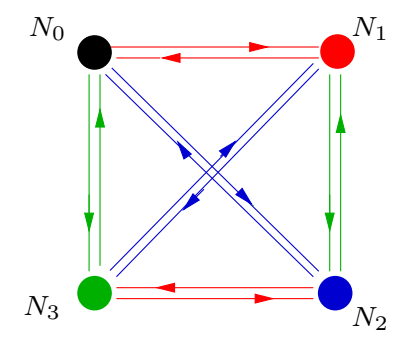

Figure 1: The quiver diagram encoding the field content and the charges for fractional D-branes of the orbifold $\mathbb{C}^{3} /\left(\mathbb{Z}_{2} \times \mathbb{Z}_{2}\right)$. The dots represent the branes associated with the irrep $R_{A}$ of the orbifold group. A stack of $N_{A}$ such branes supports a $\mathrm{U}\left(N_{A}\right)$ gauge theory. An oriented link from the $A$-th to the $B$-th dot corresponds to a chiral multiplet transforming in the $\left(N_{A}, \bar{N}_{B}\right)$ representation of the gauge group and in the $R_{A} \otimes R_{B}$ representation of the orbifold group.

\begin{tabular}{cc|cccc}
\hline \hline fields & $A_{\mu}$ & $\Lambda^{\alpha}$ & $\phi^{I}$ & $\psi^{\alpha I}$ \\
\hline charge & $q$ & 0 & $+\frac{3}{2}$ & +1 & $-\frac{1}{2}$ \\
charge & $q^{\prime}$ & 0 & 0 & $\delta_{I 1}-\delta_{I 2}$ & $\delta_{I 1}-\delta_{I 2}$ \\
charge $q^{\prime \prime}$ & 0 & 0 & $\delta_{I 1}-\delta_{I 3}$ & $\delta_{I 1}-\delta_{I 3}$ \\
\hline \hline
\end{tabular}

Table 2: The charges $q, q^{\prime}$ and $q^{\prime \prime}$ of the various fields of the $\mathcal{N}=1$ quiver gauge theory. Here $A_{\mu}$ is the gauge vector, $\Lambda^{\alpha}$ is the corresponding gaugino while $\psi^{\alpha I}$ is the fermion of the bifundamental matter superfield $\Phi^{I}$ of which $\phi^{I}$ is the lowest component. The complex conjugate fields $\bar{\phi}_{I}, \bar{\Lambda}_{\dot{\alpha}}$ and $\bar{\psi}_{\dot{\alpha} I}$ transform oppositely to the ones displayed. Finally $\theta$ transforms as $\Lambda$ and oppositely to $\bar{\theta}$.

The values of these charges for the various gauge fields are displayed in Table 2.

The fractional D3-branes can also be thought of as D5-branes wrapping exceptional (i.e. vanishing) 2 -cycles $\mathcal{C}_{A}$ of the orbifold. Note that there are only three independent such cycles on $\mathbb{C}^{3} /\left(\mathbb{Z}_{2} \times \mathbb{Z}_{2}\right)$ which are associated to the three exceptional $\mathbb{P}^{1}$ 's corresponding to the non-trivial elements $h_{I}$ of $\mathbb{Z}_{2} \times \mathbb{Z}_{2}$. This implies that only three linear combinations of the $\mathcal{C}_{A}$ 's are really independent. Indeed, the linear combination $\sum_{A=0}^{3} \mathcal{C}_{A}$ is trivial in the homological sense since a D5 brane wrapping this cycle transforms in the regular representation and can move freely away from the singularity because it is made of a D5brane plus its three images under the orbifold group. The gauge kinetic functions $\tau_{A}$ of the four $\mathrm{U}\left(N_{A}\right)$ factors can be expressed in terms of the three Kähler parameters describing the complexified string volumes of the three non-trivial independent 2-cycles and the axiondilaton field $\tau$. In the unresolved (singular) orbifold limit, which from the string point of view corresponds to switching off the fluctuations of all twisted closed string fields, we simply have $[50,11]$

$$
\tau_{A}=\frac{\theta_{A}}{2 \pi}+\mathrm{i} \frac{4 \pi^{2}}{g_{A}^{2}}=\frac{1}{4} \tau
$$

for all $A$ 's. However, by turning on twisted closed string moduli, one can introduce differences among the $\tau_{A}$ 's and thus distinguish the gauge couplings of the various group factors. 


\subsection{The instanton moduli space}

A very similar analysis applies to instantonic sectors of the gauge theory. In this framework instantons are realized with $\mathrm{D}(-1)$-branes and their moduli space is described by the lowest modes of open strings with at least one end-point on the $\mathrm{D}(-1)$-branes. The $\mathrm{U}(N)$ gauge theory dynamics in the sector with instanton number $k$ can be efficiently described by the $\alpha^{\prime} \rightarrow 0$ limit of the action of a system of $k \mathrm{D}(-1)$-branes and $N$ D3-branes on $\mathbb{C}^{3}$. This system is described in terms of a $\mathrm{U}(N) \times \mathrm{U}(k)$ matrix theory whose action is [23]

$$
S_{\mathrm{D} 3 / \mathrm{D}(-1)}=\operatorname{Tr}_{k}\left[\frac{1}{2 g_{0}^{2}} S_{G}+S_{K}+S_{D}+S_{\phi}\right]
$$

with

$$
\begin{aligned}
S_{G} & =D_{c} D^{c}-\frac{1}{2}\left[\chi_{m}, \chi_{n}\right]^{2}-\mathrm{i} \lambda_{\dot{\alpha} A}\left[\chi^{A B}, \lambda_{B}^{\dot{\alpha}}\right], \\
S_{K} & =\chi_{m} \bar{w}_{\dot{\alpha}} w^{\dot{\alpha}} \chi^{m}-\left[\chi_{m}, a_{\mu}\right]^{2}-\mathrm{i} M^{\alpha A}\left[\bar{\chi}_{A B}, M_{\alpha}^{B}\right]+\frac{\mathrm{i}}{2} \bar{\chi}_{A B} \bar{\mu}^{A} \mu^{B}, \\
S_{D} & =\mathrm{i} D_{c}\left(\bar{w}_{\dot{\alpha}}\left(\tau^{c}\right)_{\dot{\beta}}^{\dot{\alpha}} w^{\dot{\beta}}-\mathrm{i} \bar{\eta}_{\mu \nu}^{c}\left[a^{\mu}, a^{\nu}\right]\right)+\mathrm{i} \lambda_{\dot{\alpha} A}\left(\bar{\mu}^{A} w^{\dot{\alpha}}+\bar{w}^{\dot{\alpha}} \mu^{A}-\left[a^{\alpha \dot{\alpha}}, M_{\alpha}{ }^{A}\right]\right), \\
S_{\phi} & =\frac{1}{8} \epsilon^{A B C D} \bar{w}_{\dot{\alpha}} \bar{\phi}_{A B} \bar{\phi}_{C D} w^{\dot{\alpha}}+\frac{1}{2} \bar{w}_{\dot{\alpha}} \phi^{A B} w^{\dot{\alpha}} \bar{\chi}_{A B}+\frac{\mathrm{i}}{2} \bar{\mu}^{A} \bar{\phi}_{A B} \mu^{B} .
\end{aligned}
$$

The fields entering in (2.12) represent the lowest modes of open strings with $\mathrm{D}(-1) / \mathrm{D}(-1)$ and $\mathrm{D}(-1) / \mathrm{D} 3$ boundary conditions and are

$$
\mathfrak{M}=\left\{a_{\mu}, \chi_{m}, D_{c}, M^{\alpha A}, \lambda_{\dot{\alpha} A}\right\}^{i}{ }_{j} \cup\left\{w_{\dot{\alpha}}, \mu^{A}\right\}_{i}^{u} \cup\left\{\bar{w}_{\dot{\alpha}}, \bar{\mu}^{A}\right\}^{i}{ }_{u}
$$

with $i, j=1, \ldots, k$ and $u, v=1, \ldots, N$ labeling the $k \mathrm{D}(-1)$ and the $N$ D3 boundaries respectively. The other indices run over the following domains: $\mu, \nu=0, \ldots, 3 ; \alpha, \dot{\alpha}=1,2$; $m, n=1, \ldots, 6 ; A, B=0, \ldots, 3$, labeling, respectively, the vector and spinor representations of the $\mathrm{SO}(4)$ Lorentz group and of the $\mathrm{SO}(6) \sim \mathrm{SU}(4)$ internal-symmetry group ${ }^{3}$, while $c=1,2,3$. We have also defined

$$
\begin{array}{ll}
\chi^{A B}=\chi_{m}\left(\Sigma^{m}\right)^{A B}, \quad \bar{\chi}_{A B}=\chi_{m}\left(\bar{\Sigma}^{m}\right)_{A B}=\frac{1}{2} \epsilon_{A B C D} \chi^{C D}, \\
\phi^{A B}=\phi_{m}\left(\Sigma^{m}\right)^{A B} \quad, \quad \bar{\phi}_{A B}=\phi_{m}\left(\bar{\Sigma}^{m}\right)_{A B}=\frac{1}{2} \epsilon_{A B C D} \phi^{C D}
\end{array}
$$

where $\Sigma^{m}$ and $\bar{\Sigma}^{m}$ are the chiral and anti-chiral blocks of the Dirac matrices in the sixdimensional internal space, and $\phi_{m}$ are the six vacuum expectation values of the scalar fields $\Phi^{I}$ in the real basis. Finally,

$$
\frac{1}{g_{0}^{2}}=\frac{\pi}{g_{s}}\left(2 \pi \alpha^{\prime}\right)^{2}
$$

is the coupling constant of the gauge theory on the $\mathrm{D}(-1)$ branes. The scaling dimensions of the various moduli appearing in (2.12) are listed in Tab. 3.

\footnotetext{
${ }^{3}$ See footnote 2 .
} 


\begin{tabular}{c|ccccccccc}
\hline \hline moduli & $a_{\mu}$ & $\chi_{m}$ & $D_{c}$ & $M^{\alpha A}$ & $\lambda_{\dot{\alpha} A}$ & $w_{\dot{\alpha}}$ & $\bar{w}_{\dot{\alpha}}$ & $\mu^{A}$ & $\bar{\mu}^{A}$ \\
\hline dimension & $M_{s}^{-1}$ & $M_{s}$ & $M_{s}^{2}$ & $M_{s}^{-1 / 2}$ & $M_{s}^{3 / 2}$ & $M_{s}^{-1}$ & $M_{s}^{-1}$ & $M_{s}^{-1 / 2}$ & $M_{s}^{-1 / 2}$ \\
\hline \hline
\end{tabular}

Table 3: Scaling dimensions of the ADHM moduli in terms of the string scale $M_{s}=\left(2 \pi \alpha^{\prime}\right)^{-1 / 2}$.

The action (2.11) follows from dimensional reduction of the six-dimensional action of the D5/D9 brane system down to zero dimensions and, as discussed in detail in Ref. [18], it can be explicitly derived from scattering amplitudes of open strings with $\mathrm{D}(-1) / \mathrm{D}(-1)$ or $\mathrm{D}(-1) / \mathrm{D} 3$ boundary conditions on (mixed) disks. In the field theory limit $\alpha^{\prime} \rightarrow 0$ (i.e. $\left.g_{0} \rightarrow \infty\right)$, the term $S_{G}$ in (2.11) can be discarded and the fields $D^{c}$ and $\lambda_{\dot{\alpha} A}$ become Lagrange multipliers for the super ADHM constraints that realize the D- and F-flatness conditions in the matrix theory.

Now let us consider the $\mathbb{Z}_{2} \times \mathbb{Z}_{2}$ orbifold projection. The group $\mathrm{U}(N) \times \mathrm{U}(k)$ breaks down to $\prod_{A} \mathrm{U}\left(N_{A}\right) \times \mathrm{U}\left(k_{A}\right)$ with $N_{A}$ and $k_{A}$ being the numbers of fractional D3 and $\mathrm{D}(-1)$ branes of type $A$ such that

$$
N=\sum_{A=0}^{3} N_{A} \quad \text { and } \quad k=\sum_{A=0}^{3} k_{A} .
$$

Consequently, the indices $u$ and $i$ break into $u_{A}=1, \ldots, N_{A}$ and $i_{A}=1, \ldots, k_{A}$, while the spinor index $A$ splits into $A=(0, I)$ with $A=0$ denoting the $\mathcal{N}=1$ unbroken symmetry. For the sake of simplicity from now on we always omit the index 0 and write

$$
M^{\alpha 0} \equiv M^{\alpha} \quad, \quad \lambda_{\dot{\alpha} 0} \equiv \lambda_{\dot{\alpha}} \quad, \quad \mu^{0} \equiv \mu \quad, \quad \bar{\mu}^{0} \equiv \bar{\mu} .
$$

Furthermore we set

$$
\begin{aligned}
\bar{\chi}_{0 I} & \equiv \bar{\chi}_{I}, & \chi^{0 I} & =\frac{1}{2} \epsilon^{I J K} \bar{\chi}_{J K} \equiv \chi^{I}, \\
\bar{\phi}_{0 I} & \equiv \bar{\phi}_{I}=\left\langle\bar{\Phi}_{I}\right\rangle & \phi^{0 I} & =\frac{1}{2} \epsilon^{I J K} \bar{\phi}_{J K} \equiv \phi^{I}=\left\langle\Phi^{I}\right\rangle .
\end{aligned}
$$

This notation makes more manifest which zero-modes couple to the holomorphic superfields and which others couple to the anti-holomorphic ones. Indeed, the action $S_{\phi}$ in $(2.12)$ becomes

$$
\begin{aligned}
S_{\phi}= & \frac{1}{2} \bar{w}_{\dot{\alpha}}\left(\phi^{I} \bar{\phi}_{I}+\bar{\phi}_{I} \phi^{I}\right) w^{\dot{\alpha}}+\bar{w}_{\dot{\alpha}} \phi^{I} w^{\dot{\alpha}} \bar{\chi}_{I}+\chi^{I} \bar{w}_{\dot{\alpha}} \bar{\phi}_{I} w^{\dot{\alpha}} \\
& -\frac{\mathrm{i}}{2} \bar{\mu} \bar{\phi}_{I} \mu^{I}+\frac{\mathrm{i}}{2} \bar{\mu}^{I} \bar{\phi}_{I} \mu-\frac{\mathrm{i}}{2} \epsilon_{I J K} \bar{\mu}^{I} \phi^{J} \mu^{K} .
\end{aligned}
$$

Taking into account the $\mathbb{Z}_{2} \times \mathbb{Z}_{2}$ transformation properties of the various fields and of their Chan-Paton labels, one finds that the moduli that survive the orbifold projection are

$$
\begin{aligned}
\mathfrak{M}= & \left\{a_{\mu}, D_{c}, M^{\alpha}, \lambda_{\dot{\alpha}}\right\}_{j_{A}}^{i_{A}} \cup\left\{w_{\dot{\alpha}}, \mu\right\}_{j_{A}}^{u_{A}} \cup\left\{\bar{w}_{\dot{\alpha}}, \bar{\mu}\right\}_{u_{A}}^{i_{A}} \\
& \cup\left\{\chi^{I}, \bar{\chi}_{I}, M^{\alpha I}, \lambda_{\dot{\alpha} I}\right\}_{j_{A \otimes I}}^{i_{A}} \cup\left\{\mu^{I}\right\}_{i_{A \otimes I}}^{u_{A}} \cup\left\{\bar{\mu}^{I}\right\}_{u_{A \otimes I}}^{i_{A}} .
\end{aligned}
$$

Like for the chiral multiplets in (2.6), the non-trivial transformation of the instanton moduli carrying an index $I$ is compensated by a similar transformation of the Chan-Paton labels 
making the whole expression invariant under the orbifold group, as indicated in the second line of $(2.20)$.

The $\left(\mathbb{Z}_{2} \times \mathbb{Z}_{2}\right)$ projected moduli action is invariant under the $\mathrm{U}(1)^{3} \subset \mathrm{SO}(6)$ symmetry whose properties on the D3/D3 sector we already discussed in Sec. 2.1, see Table 2. The charges of the moduli with respect to the same choice of $\mathrm{U}(1)^{3}$ made in Eq. (2.9) are given in Table 4.

\begin{tabular}{|c|c|c|c|c|c|c|}
\hline moduli & $\chi^{I}$ & $\bar{\chi}_{I}$ & $\mu, \bar{\mu}, M^{\alpha}$ & $\mu^{I}, \bar{\mu}^{I}, M^{\alpha I}$ & $\lambda_{\dot{\alpha}}$ & $\lambda_{\dot{\alpha} I}$ \\
\hline charge $q$ & +1 & -1 & $+\frac{3}{2}$ & $-\frac{1}{2}$ & $-\frac{3}{2}$ & $+\frac{1}{2}$ \\
\hline charge $q^{\prime}$ & $\delta_{I 1}-\delta_{I 2}$ & $\delta_{I 2}-\delta_{I 1}$ & 0 & $\delta_{I 1}-\delta_{I 2}$ & 0 & $\delta_{I 2}-\delta_{I 1}$ \\
\hline charge $q^{\prime \prime}$ & $\delta_{I 1}-\delta_{I 3}$ & $\delta_{I 3}-\delta_{I 1}$ & 0 & $\delta_{I 1}-\delta_{I 3}$ & 0 & $\delta_{I 3}-\delta_{I 1}$ \\
\hline
\end{tabular}

Table 4: The charges $q, q^{\prime}$ and $q^{\prime \prime}$ of the various fields of the $\mathrm{D} 3 / \mathrm{D}(-1)$ brane system. The bosonic moduli $a^{\mu}, w_{\dot{\alpha}}, \bar{w}_{\dot{\alpha}}$ and $D_{c}$ are neutral under all three $\mathrm{U}(1)$ 's.

Among the moduli in $\mathfrak{M}$, the bosonic combinations

$$
x^{\mu} \equiv \frac{1}{k} \sum_{A=0}^{3} \sum_{i_{A}=1}^{k_{A}}\left\{a^{\mu}\right\}_{i_{A}}^{i_{A}}
$$

represent the center of mass coordinates of the $\mathrm{D}(-1)$-branes and can be interpreted as the Goldstone modes associated to the translational symmetry of the D3-branes that is broken by the D-instantons. Thus they can be identified with the space-time coordinates, and indeed have dimensions of a length. Similarly, the fermionic combinations

$$
\theta^{\alpha} \equiv \frac{1}{k} \sum_{A=0}^{3} \sum_{i_{A}=1}^{k_{A}}\left\{M^{\alpha}\right\}_{i_{A}}^{i_{A}}
$$

are the Goldstinos for the two supersymmetries of the D3-branes that are broken by the $\mathrm{D}(-1)$-branes, and thus they can be identified with the chiral fermionic superspace coordinates. Indeed they have dimensions of (length) ${ }^{1 / 2}$. Notice that neither $x^{\mu}$ nor $\theta^{\alpha}$ appear in the moduli action obtained by projecting (2.11).

The moduli (2.20) account for both gauge and stringy instantons. Gauge instantons correspond to $\mathrm{D}(-1)$-branes that sit on non-empty nodes of the quiver diagram, so that their number $k_{A}$ can be interpreted as the second Chern class of the Yang-Mills bundle of the $\mathrm{U}\left(N_{A}\right)$ component of the gauge group. Stringy instantons correspond instead to $\mathrm{D}(-1)$ branes occupying empty nodes of the quiver, so that in this case we can set $k_{A} N_{A}=0$ for all $A$ 's. From (2.20) one sees that in the stringy instanton case the modes $w_{\dot{\alpha}}, \bar{w}_{\dot{\alpha}}, \mu$ and $\bar{\mu}$ are missing since there are no $k_{A} \times N_{A}$ invariant blocks and thus the only moduli are the charged fermionic fields $\mu^{I}$ and $\bar{\mu}^{I}$. Recalling that the bosonic $w$-moduli describe the instanton sizes and gauge orientations, one can say that exotic instantons are entirely specified by their spacetime positions. The presence (absence) of bosonic modes in the $\mathrm{D} 3 / \mathrm{D}(-1)$ sector for gauge (stringy) instantons can be understood in geometric terms by 
blowing up 2-cycles at the singularity and reinterpreting the fractional $\mathrm{D} 3 / \mathrm{D}(-1)$ system in terms of a D5/E1 bound state wrapping an exceptional 2-cycle of $\mathbb{C}^{3} /\left(\mathbb{Z}_{2} \times \mathbb{Z}_{2}\right)$. Gauge (stringy) instantons correspond to the cases when the D5 brane and the E1 are (are not) parallel in the internal space. In the first case the number of Neumann-Dirichlet directions is 4 and therefore the NS ground states is massless. In the stringy case, the number of Neumann-Dirichlet directions exceeds 4 and therefore the NS ground state is massive and all charged moduli come only from the fermionic $\mathrm{R}$ sector.

\section{D-instanton partition function}

In this section we study the non-perturbative effects generated by fractional D-instantons on the $\mathcal{N}=1$ gauge theory realized with space-filling fractional D3 branes. For simplicity from now on we take (see Fig. 2)

$$
N_{2}=N_{3}=0 \quad \text { with } \quad N_{0} \text { and } N_{1} \text { arbitrary , }
$$

which is the simplest configuration that allows us to discuss both gauge and stringy instanton effects.

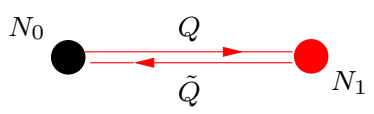

Figure 2: This simple quiver gauge theory is (from the point of view of one of the nodes) just $\mathcal{N}=1 \mathrm{SQCD}$.

This brane system describes a $\mathcal{N}=1$ theory with gauge group $\mathrm{U}\left(N_{0}\right) \times \mathrm{U}\left(N_{1}\right)$ and a single bifundamental multiplet

$$
\Phi^{1}(x, \theta) \equiv \Phi(x, \theta)=\phi(x)+\sqrt{2} \theta \psi(x)+\theta^{2} F(x),
$$

which in block form is

$$
\Phi=\left(\begin{array}{cc}
0 & Q_{f}^{u} \\
\widetilde{Q}_{u}^{f} & 0
\end{array}\right)
$$

with $u=1, \ldots N_{0}$ and $f=1, \ldots N_{1}$. The two off-diagonal blocks $Q$ and $\widetilde{Q}$ represent the quark and anti-quark superfields which transform respectively in the fundamental and anti-fundamental of $\mathrm{U}\left(N_{0}\right)$, and in the anti-fundamental and fundamental of $\mathrm{U}\left(N_{1}\right)$. Both quarks and anti-quarks are neutral under the diagonal $\mathrm{U}(1)$ factor of the gauge group, which decouples. On the other hand [51] the relative $\mathrm{U}(1)$ group, under which both $Q$ and $\widetilde{Q}$ are charged, is IR free and thus at low energies the resulting effective gauge group is $\mathrm{SU}\left(N_{0}\right) \times \mathrm{SU}\left(N_{1}\right)$. Therefore, from the point of view of, say, the $\mathrm{SU}\left(N_{0}\right)$ factor this theory is just $\mathcal{N}=1 \mathrm{SQCD}$ with $N_{c}=N_{0}$ colors and $N_{f}=N_{1}$ flavors. In the following we will study the non-perturbative properties of this theory in the Higgs phase where the gauge invariance is completely broken by giving (large) vacuum expectation values to the lowest components of the matter superfields. This requires $N_{f} \geq N_{c}-1$. The moduli space of 
this SQCD is obtained by imposing the D-flatness conditions. As remarked in [52], even if the effective gauge group is $\mathrm{SU}\left(N_{c}\right)$, we have to impose the D-term equations also for the (massive) ${ }^{4} \mathrm{U}(1)$ factors to obtain the correct moduli space of the quiver theory; in our case these D-term conditions lead to the constraint

$$
Q \bar{Q}-\overline{\widetilde{Q}} \widetilde{Q}=\xi \mathbb{1}_{N_{c} \times N_{c}}
$$

where $\xi$ is a Fayet-Iliopoulos parameter related to twisted closed string fields which vanish in the singular orbifold limit. For $N_{f} \geq N_{c}$ the D-term constraints allow for flat directions parameterized by meson fields

$$
M_{f_{2}}^{f_{1}} \equiv \widetilde{Q}_{u}^{f_{1}} Q_{f_{2}}^{u}
$$

and baryon fields

$$
B_{f_{1} \ldots f_{N_{c}}}=\epsilon_{u_{1} \ldots u_{N_{c}}} Q_{f 1}^{u_{1}} \ldots Q_{f_{N_{c}}}^{u_{N_{c}}} \quad, \quad \widetilde{B}^{f_{1} \ldots f_{N_{c}}}=\epsilon^{u_{1} \ldots u_{N_{c}}} \widetilde{Q}_{u_{1}}^{f 1} \ldots \widetilde{Q}_{u_{N_{c}}}^{f_{N_{c}}}
$$

which are subject to constraints whose specific form depends on the difference $\left(N_{f}-N_{c}\right)$ (see for instance Ref. [53]). These are the good observables of the low-energy theory in the Higgs phase. For $N_{f}=N_{c}-1$, instead, the baryons cannot be formed and only the meson fields are present.

To have a quick understanding of the non-perturbative effects that can be obtained in our stringy set-up, it is convenient to use dimensional analysis and exploit the symmetries of the $\mathrm{D} 3 / \mathrm{D}(-1)$ brane system; we will see that besides the well-known one-instanton effects like the ADS superpotential at $N_{f}=N_{c}-1$ [49], in the quiver theory an infinite tower of multi-instanton corrections to the superpotential are in principle allowed. This is what we are going to show in the remainder of this section. In Section 4 we specialize our discussion to the one-instanton sector and, using again dimensional analysis and symmetry considerations, we analyze various types of non-perturbative effects in the low-energy theory, as a preparation for the study of the flux-induced terms presented in Sections 5 and 6.

\subsection{The moduli space integral}

The non-perturbative effects produced by a configuration of fractional D-instantons with numbers $k_{A}$ can be analyzed by studying the centered partition function

$$
W_{\text {n.p. }}=\int d \widehat{\mathfrak{M}} \prod_{A=0}^{3}\left(M_{s}^{k_{A} \beta_{A}} \mathrm{e}^{2 \pi \mathrm{i} k_{A} \tau_{A}} \mathrm{e}^{-\operatorname{Tr}_{k_{A}}\left[S_{K}+S_{D}+S_{\phi}\right]}\right)
$$

where the integration is over all moduli listed in (2.20) except for the center of mass supercoordinates $x^{\mu}$ and $\theta^{\alpha}$ defined in (2.21) and (2.22). These centered moduli are collectively denoted by $\widehat{\mathfrak{M}}$. The action $\operatorname{Tr}_{k_{A}}\left[S_{K}+S_{D}+S_{\phi}\right]$ is obtained by taking the field theory limit in (2.11) and restricting the moduli to their $\mathbb{Z}_{2} \times \mathbb{Z}_{2}$ invariant blocks for each $A$, while the term $2 \pi \mathrm{i} k_{A} \tau_{A}$ represents the classical action of $k_{A}$ fractional D-instantons of type $A$ (see

\footnotetext{
${ }^{4}$ For a discussion of this point in the same orbifold model we are considering, see for example the beginning of Section 3 of the published version of Ref. [54].
} 
Eqs. (1.4) and (2.10)). Finally the power of the string scale $M_{s}$ compensates for the scaling dimensions of the measure over the centered moduli space so that the centered partition function $W_{\text {n.p. }}$ has mass dimension 3, as expected. Indeed, using Tab. 3 one can easily show that the mass dimension $D$ of the instanton measure is

$$
D[d \widehat{\mathfrak{M}}]=3-\sum_{A=0}^{3} k_{A} \beta_{A}
$$

where $\beta_{A}$ is the one-loop $\beta$-function coefficient of the $\mathcal{N}=1 \mathrm{SU}\left(N_{A}\right)$ gauge theory with $\sum_{I=1}^{3} N_{A \otimes I}$ fundamentals and anti-fundamentals, namely

$$
\beta_{A}=3 \ell\left(\operatorname{Adj}_{A}\right)-2 \sum_{I=1}^{3} N_{A \otimes I} \ell\left(N_{A}\right)=3 N_{A}-\sum_{I=1}^{3} N_{A \otimes I}
$$

where $\ell(r)$ denotes the index ${ }^{5}$ of the representation $r$. It is interesting to remark that the explicit expression of $\beta_{A}$ is well-defined even in the case $N_{A}=0$ where it cannot be interpreted as the $\beta$-function coefficient of any gauge theory. Keeping this in mind, all formulas in this section can be applied to both gauge and stringy instanton configurations.

Coming back to the centered partition function (3.7) one can ask which dependence on the scalar vacuum expectation values is generated by the integral over the instanton moduli. A quick answer to this question follows by requiring that the form of $W_{\text {n.p. }}$ be consistent with the symmetries of the $\mathrm{D} 3 / \mathrm{D}(-1)$ system. In particular we can exploit the $\mathrm{U}(1)^{3}$ symmetries left unbroken by the orbifold projection that we have discussed in Section 2. These are symmetries of the $\mathrm{D} 3 / \mathrm{D}(-1)$ action but not of the instanton measure. Indeed, since there are unpaired moduli, like $\mu^{A}$ and $\bar{\mu}^{A}$, which transform in the same way under $U(1)^{3}$, the charges of the centered instanton measure, and hence of $W_{\text {n.p. }}$, are non-trivial. In particular, the charge $q$ is ${ }^{6}$

$$
q[d \widehat{\mathfrak{M}}]=-2 n_{\mu} q(\mu)-2 n_{\mu^{I}} q\left(\mu^{I}\right)-2 q(\lambda)
$$

where $n_{\mu}$ and $n_{\mu^{I}}$ are the numbers of $\mu$ and $\mu^{I}$ moduli, and the factors of 2 account for identical contributions from $\bar{\mu}$ and $\bar{\mu}^{I}$. Finally, the term $-2 q(\lambda)$ comes from the two components of the anti-chiral fermion

$$
\lambda_{\dot{\alpha}} \equiv \frac{1}{k} \sum_{A=0}^{3} \sum_{i_{A}=1}^{k_{A}}\left\{\lambda_{\dot{\alpha}}\right\}_{i_{A}}^{i_{A}}
$$

which are unpaired since their partners, namely the fermionic superspace coordinates $\theta^{\alpha}$ defined in (2.22), have been taken out from the centered measure $d \widehat{\mathfrak{M}}$. The minus signs

\footnotetext{
${ }^{5}$ The index $\ell(r)$ is defined by $\operatorname{Tr}_{r}\left(T^{a} T^{b}\right)=\ell(r) \delta^{a b}$. For $\mathrm{SU}(N)$ gauge groups, the indices in the adjoint, fundamental, symmetric and antisymmetric representations are, respectively, given by $\ell(\operatorname{Adj})=N$, $\ell(N)=\frac{1}{2}, \ell\left(\frac{1}{2} N(N+1)\right)=N+2$ and $\ell\left(\frac{1}{2} N(N-1)\right)=N-2$.

${ }^{6}$ As one can see from Tab. 4 , the moduli $M^{\alpha I}$ and $\lambda_{\dot{\alpha} I}$ have opposite charges, like $M^{\alpha}$ and $\lambda_{\dot{\alpha}}$. For this reason they do not contribute to the total charges of the centered measure $d \widehat{\mathfrak{M}}$ which thus depend only on the charges of unpaired moduli.
} 
in (3.10) come from the fact that a fermionic differential transforms oppositely to the field itself. Using the charges listed in Tab. 4, it is easy to rewrite (3.10) as

$$
q[d \widehat{\mathfrak{M}}]=3-\sum_{A=0}^{3} k_{A}\left(3 N_{A}-\sum_{I=1}^{3} N_{A \otimes I}\right)=3-\sum_{A=0}^{3} k_{A} \beta_{A} .
$$

In a similar way one finds

$$
\begin{gathered}
q^{\prime}[d \widehat{\mathfrak{M}}]=-2 n_{\mu} q^{\prime}(\mu)-2 n_{\mu^{I}} q^{\prime}\left(\mu^{I}\right)-2 q^{\prime}(\lambda)=-2 \sum_{A=0}^{3} k_{A}\left(N_{A \otimes 1}-N_{A \otimes 2}\right) \\
q^{\prime \prime}[d \widehat{\mathfrak{M}}]=-2 n_{\mu} q^{\prime \prime}(\mu)-2 n_{\mu^{I}} q^{\prime \prime}\left(\mu^{I}\right)-2 q^{\prime \prime}(\lambda)=-2 \sum_{A=0}^{3} k_{A}\left(N_{A \otimes 1}-N_{A \otimes 3}\right) .
\end{gathered}
$$

One can check that the $\mathrm{U}(1)^{3}$ charges of the ADHM measure coincide with the ones of the moduli space of instanton zero-modes. In fact, since in an instanton background the bosonic zero-modes always come together with their complex conjugates, the charges $q$, $q^{\prime}$ and $q^{\prime \prime}$ of the instanton measure depend only on the number of fermionic zero-modes, namely on the number $n_{\Lambda}$ of gaugino zero-modes, and on the number $n_{\psi^{I}}$ of zero-modes of the fundamental matter fields ${ }^{7}$. These numbers are given by the index of the Dirac operator evaluated, respectively, in the adjoint and fundamental $\mathrm{SU}\left(N_{A}\right)$ representations under which the fields transform, i.e.

$$
\begin{aligned}
n_{\Lambda} & =2 k_{A} \ell\left(\operatorname{Adj}_{A}\right)=2 k_{A} N_{A}, \\
n_{\psi^{I}} & =2 k_{A}\left(2 N_{A \otimes I}\right) \ell\left(N_{A}\right)=2 k_{A} N_{A \otimes I} .
\end{aligned}
$$

Taking into account the contribution of the two fermionic superspace coordinates $\theta^{\alpha}$ to the charges of the instanton measure and using the values reported in Tab. 2, we have

$$
\begin{aligned}
q & =-n_{\Lambda} q(\Lambda)-\sum_{I=1}^{3} n_{\psi^{I}} q\left(\psi^{I}\right)-2 q(\theta)=-\frac{3}{2} n_{\Lambda}+\frac{1}{2} \sum_{I=1}^{3} n_{\psi^{I}}+3 \\
q^{\prime} & =-n_{\Lambda} q^{\prime}(\Lambda)-\sum_{I=1}^{3} n_{\psi^{I}} q^{\prime}\left(\psi^{I}\right)-2 q^{\prime}(\theta)=-n_{\psi^{1}}+n_{\psi^{2}}, \\
q^{\prime \prime} & =-n_{\Lambda} q^{\prime \prime}(\Lambda)-\sum_{I=1}^{3} n_{\psi^{I}} q\left(\psi^{I}\right)-2 q^{\prime \prime}(\theta)=-n_{\psi^{1}}+n_{\psi^{3}} .
\end{aligned}
$$

Exploiting (3.14), it is immediate to see that these charges coincide with the ones given in (3.12) and (3.13) and computed using the ADHM construction.

So far we have considered a generic D-instanton configuration. From now on we will focus on two cases, namely gauge and stringy instantons, which correspond to the following choices of $k_{A}$ 's

$$
\begin{aligned}
\text { gauge : } & \left(k_{0}, k_{1}, 0,0\right), \\
\text { stringy : } & \left(0,0, k_{2}, k_{3}\right) .
\end{aligned}
$$


a)

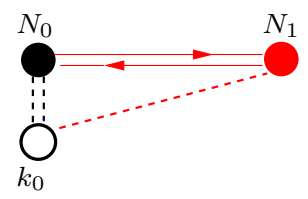

b)

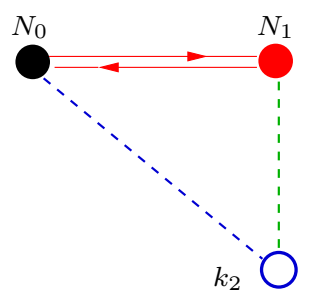

Figure 3: D3/D(-1)-quiver for SQCD with a) gauge instantons and b) stringy instantons. Filled and empty circles represent stacks of D3 and D $(-1)$ branes, solid lines stand for chiral bifundamental matter, dashed lines for charged instanton moduli. A single dashed line represents the fermions $\mu$, while a double dashed line is a $(\mu, w)$ pair.

Some gauge and stringy instanton quiver diagrams are displayed in Fig. 3.

As noticed above, for stringy instantons we have $k_{A} N_{A}=0$ and therefore the only charged ADHM moduli that survive are $\mu^{I}$ and $\bar{\mu}^{I}$, while $w, \bar{w}, \mu$ and $\bar{\mu}$ are absent. As a consequence, the fermion $\lambda$ of Eq. (3.11) decouples from the moduli action and in the centered partition function of stringy instantons there is an unbalanced fermionic zero-mode integration. Therefore, unless such zero-modes are removed, for example with an orientifold projection $[28,29,30]$, or lifted with some mechanism [39, 45], one gets a vanishing result. We will return to the stringy instanton configurations in Section 5 where we discuss how bulk fluxes can cure this problem. In the remaining part of this section we instead analyze in more detail the instanton partition function for a generic configuration of gauge instantons.

\subsection{Zero-mode counting for gauge instantons}

For gauge instantons the centered partition function (3.7) is a function of $\phi$ and $\bar{\phi}$, i.e. of the vacuum expectation values of the matter superfield (3.3) and its conjugate, with scaling dimension 3 . The most general ansatz for $W_{\text {n.p. }}$ is therefore

$$
W_{\text {n.p. }}=\mathcal{C} M_{s}^{\left(k_{0} \beta_{0}+k_{1} \beta_{1}\right)} \mathrm{e}^{2 \pi \mathrm{i}\left(k_{0} \tau_{0}+k_{1} \tau_{1}\right)} \bar{\phi}^{n} \phi^{m}
$$

with $n+m+k_{0} \beta_{0}+k_{1} \beta_{1}=3$ and $\mathcal{C}$ a numerical constant. Using Tab. 2, it is easy to see that the $\mathrm{U}(1)^{3}$ charges of this expression are all equal and given by

$$
q\left[W_{\text {n.p. }}\right]=q^{\prime}\left[W_{\text {n.p. }}\right]=q^{\prime \prime}\left[W_{\text {n.p. }}\right]=3-2 n-k_{0} \beta_{0}-k_{1} \beta_{1} \text {. }
$$

We must require that these charges match those of the centered measure, which, as follows from (3.12) and (3.13), in this case are given by

$$
q[d \widehat{\mathfrak{M}}]=3-k_{0} \beta_{0}-k_{1} \beta_{1} \quad, \quad q^{\prime}[d \widehat{\mathfrak{M}}]=q^{\prime \prime}[d \widehat{\mathfrak{M}}]=-2 k_{0} N_{1}-2 k_{1} N_{0} .
$$

Then we immediately find that $n=0$ and

$$
\left(k_{0}-k_{1}\right)\left(N_{0}-N_{1}\right)=1 .
$$

\footnotetext{
${ }^{7}$ Remember that in an instanton background $\bar{\Lambda}=\bar{\psi}_{I}=0$
} 
This equation is solved by

$$
k_{1}=k_{0}-1 \quad, \quad N_{1}=N_{0}-1
$$

or by

$$
k_{0}=k_{1}-1 \quad, \quad N_{0}=N_{1}-1 .
$$

Thus the partition function generated by gauge instantons is, as expected, an holomorphic function of $\phi$ and is given by

$$
W_{\text {n.p. }}=\mathcal{C} M_{s}^{\left(k_{0} \beta_{0}+k_{1} \beta_{1}\right)} \mathrm{e}^{2 \pi \mathrm{i}\left(k_{0} \tau_{0}+k_{1} \tau_{1}\right)} \phi^{\left(3-k_{0} \beta_{0}-k_{1} \beta_{1}\right)} .
$$

Notice that this is a formal expression in which $\phi$ stands for the vacuum expectation values of either the quark or anti-quark superfields $Q$ and $\widetilde{Q}$ defined in (3.3), and actually only the appropriate gauge invariant combinations of these should appear in the final result. The solutions above with $\left(k_{0}=1, k_{1}=0\right)$ and $\left(k_{0}=0, k_{1}=1\right)$ reproduce the well-known ADS superpotential [49] for $\mathrm{SU}\left(N_{0}\right)$ and $\mathrm{SU}\left(N_{1}\right)$ SQCD's respectively. The multi-instanton corrections with $k_{0}, k_{1}>0$ are instead a distinct feature of the quiver gauge theory we have engineered with the fractional D3 branes. We conclude by observing that we could have arrived at the same results without referring to the charges of the ADHM moduli but using instead those of the gauge field zero-modes in the instanton background.

It is possible to generalize the previous analysis of the instanton partition function by including a dependence on the entire matter fields and not only on their vacuum expectation values $\phi$ and $\bar{\phi}$. This leads to a very rich structure of non-perturbative interactions that include the holomorphic ADS superpotential when $N_{f}=N_{c}-1$ and the multi-fermion F-terms of the Beasley-Witten (BW) type [55] when $N_{f} \geq N_{c}$, plus their possible multi-instanton extensions. In the following sections we will analyze in detail such non-perturbative effective interactions in the one-instanton case.

\section{Effective interactions from gauge instantons}

In this section we discuss the non-perturbative effective interactions induced by instantons using the explicit string construction of the ADHM moduli provided by fractional D3 and $\mathrm{D}(-1)$ branes, and show that in the field theory limit $\alpha^{\prime} \rightarrow 0$ we recover the known non-perturbative F-terms, such as the ADS superpotential [49] and the BW multi-fermion couplings [55]. From now on we will consider one-instanton effects in the quiver gauge theory corresponding to a D3-brane system with $N_{2}=N_{3}=0$; this should not be regarded as a limitation of our procedure but only a choice made for the sake of simplicity.

\subsection{The gauge instanton action}

To discuss the D-instanton induced effective action on the D3 brane volume in the Higgs branch, we first have to generalize the results of Section 2.2 and introduce in the moduli action a dependence on the entire matter superfields and not only on their vacuum expectation values. As discussed in detail in [17, 18, 19], the couplings of the matter fields with the ADHM instanton moduli can be obtained by computing mixed disk diagrams with 

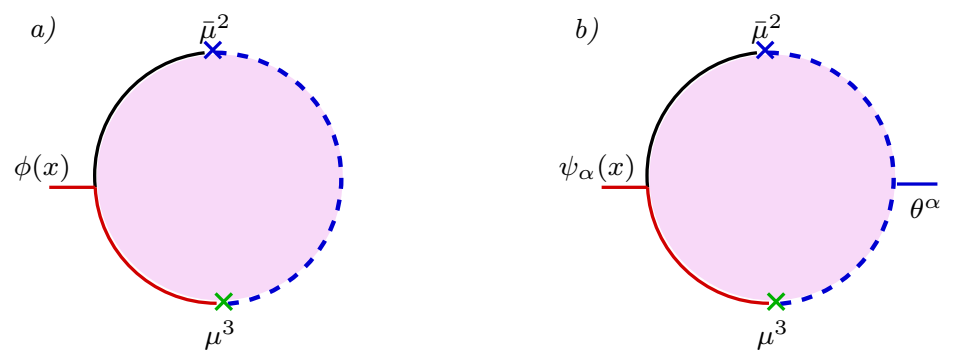

Figure 4: Disk diagrams leading to the interaction between the scalar $\phi(x)$, or its superpartner $\psi_{\alpha}(x)$, and the fermionic instanton moduli $\mu^{3}$ and $\bar{\mu}^{2}$.

insertions of vertex operators for dynamical $3 / 3$ strings on the portion of the boundary attached to the D3-branes.

An example of a coupling of $\phi(x)$ with the fermionic moduli $\mu$ and $\bar{\mu}$ is provided by the diagram of Fig. 4a), whose explicit evaluation leads to

$$
\frac{\mathrm{i}}{2} \bar{\mu}^{2} \phi(x) \mu^{3} .
$$

If $\phi(x)$ is frozen to its vacuum expectation value, this coupling precisely accounts for one of the last terms of $S_{\phi}$ given in (2.19), once we specify our D3 brane configuration ${ }^{8}$. Another possible diagram, represented in Fig. $4 b$ ), gives rise to the following coupling:

$$
-\frac{\mathrm{i}}{\sqrt{2}} \theta^{\alpha} \bar{\mu}^{2} \psi_{\alpha}(x) \mu^{3}
$$

As discussed in $[17,18,19]$, diagrams like those in Fig. 4a) and b) are related to each other by the action of the two supersymmetries of the D-instanton broken by the D3 branes. A further application of these supersymmetries leads to

$$
\frac{\mathrm{i}}{2} \theta^{2} \bar{\mu}^{2} F(x) \mu^{3}
$$

where $F$ is the auxiliary field of the matter multiplet. Also this coupling arises from a mixed disk diagram with two $\theta$-insertions on the $\mathrm{D}(-1)$ boundary and one insertion of $F$ on the D3 boundary ${ }^{9}$. Adding the contributions (4.1), (4.2) and (4.3), we reconstruct the combination

$$
\phi(x)+\sqrt{2} \theta^{\alpha} \psi_{\alpha}(x)+\theta^{2} F(x)
$$

which is the component expansion of the matter chiral superfield $\Phi(x, \theta)$ of our SQCD model. Proceeding systematically in this way, one can show that the same pattern appears

\footnotetext{
${ }^{8} \mathrm{As}$ is clear from Fig. 4, this contribution is present only when the instanton is of stringy nature, i.e. $k_{2}=1$ or $k_{3}=1$, since the $\mathrm{D}(-1)$ boundary must be of a different type with respect to the D3 boundaries. This type of contributions will play a crucial rôle in Section 6 , but we discuss it here to illustrate in a simple example how the couplings with the holomorphic matter superfields can be obtained.

${ }^{9}$ For details on the calculations of disk amplitudes involving auxiliary fields in this orbifold model see for example [56].
} 
everywhere, so that we can simply promote the vacuum expectation value $\phi$ to the complete superfield $\Phi(x, \theta)$, i.e. perform in the action (2.19) the following replacement:

$$
\phi \rightarrow \Phi(x, \theta)
$$

in order to obtain all instanton couplings with the holomorphic scalar and its superpartners.

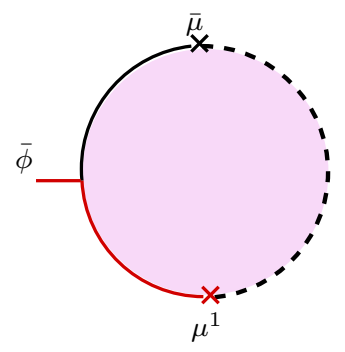

Figure 5: An example of a disk interaction between the (anti-holomorphic) scalar $\bar{\phi}(x)$ and the instanton moduli leading to the coupling (4.6).

Let us now turn to the anti-holomorphic variables. An example of a mixed disk amplitude involving the scalar $\bar{\phi}(x)$ is represented in Fig. 5. It accounts for the coupling

$$
-\frac{\mathrm{i}}{2} \bar{\mu} \bar{\phi}(x) \mu^{1},
$$

which is the obvious generalization of the first term in the second line of (2.19) when the anti-holomorphic vacuum expectation value $\bar{\phi}$ is promoted to a dynamical field. The same pattern occurs in all terms involving the anti-holomorphic vacuum expectation values $\bar{\phi}$, so that we can promote the latter with the replacement

$$
\bar{\phi} \rightarrow \bar{\phi}(x)=\left.\bar{\Phi}(x, \bar{\theta})\right|_{\bar{\theta}=0} .
$$

Notice that no $\bar{\theta}$ dependence arises, due to the half-BPS nature of the $\mathrm{D} 3 / \mathrm{D}(-1)$ system.

When one considers dynamical gauge fields, there are new types of mixed disk amplitudes that correspond to couplings which do not depend on the vacuum expectation values of the scalars; since they are not present in the action (2.19), they cannot be obtained with the replacements (4.5) and (4.7). These new types of interactions typically involve the D3/D3 anti-chiral fermions $\bar{\psi}_{\dot{\alpha}}(x)$ and correspond to the following couplings:

$$
\mathrm{i} \bar{w}_{\dot{\alpha}} \bar{\psi}^{\dot{\alpha}}(x) \mu^{1}-\mathrm{i} \bar{\mu}^{1} \bar{\psi}_{\dot{\alpha}}(x) w^{\dot{\alpha}} .
$$

Fig. 6a) represents the disk diagram corresponding to the first term of (4.8). Furthermore, using the D3 supersymmetries that are broken by the $\mathrm{D}(-1)$ branes, we can produce the following terms:

$$
-\theta_{\alpha} \bar{w}_{\dot{\beta}}\left(\bar{\sigma}^{\mu}\right)^{\alpha \dot{\beta}} \partial_{\mu} \bar{\phi}(x) \mu^{1}+\theta^{\alpha} \bar{\mu}^{1}\left(\bar{\sigma}^{\mu}\right)_{\alpha \dot{\beta}} \partial_{\mu} \bar{\phi}(x) w^{\dot{\beta}} .
$$



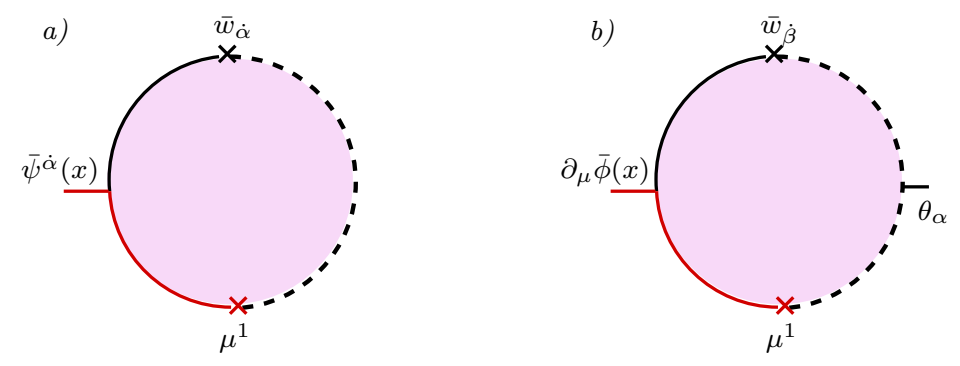

Figure 6: Examples of disk diagrams responsible for the coupling of the superfield $\left.D_{\dot{\alpha}} \bar{\Phi}(x, \bar{\theta})\right|_{\bar{\theta}=0}$ to the instanton moduli. In $b$ ) the vertex for the scalar $\bar{\phi}$ is in the 0 -th superghost picture, which leads to a derivative coupling.

The diagram responsible for the first term in (4.9) is represented in Fig. 6b). The couplings (4.9) can be obtained from (4.8) by means of the replacement

$$
\left.\bar{\psi}_{\dot{\alpha}}(x) \rightarrow \bar{D}_{\dot{\alpha}} \bar{\Phi}(x, \bar{\theta})\right|_{\bar{\theta}=0},
$$

where $\bar{D}_{\dot{\alpha}}$ is the standard spinor covariant derivative . Note that $\left.\bar{D}_{\dot{\alpha}} \bar{\Phi}(x, \bar{\theta})\right|_{\bar{\theta}=0}$ is a chiral superfield ${ }^{10}$.

We are now in the position of writing the action for the $\mathrm{D} 3 / \mathrm{D}(-1)$ system in presence of dynamical bi-fundamental matter fields, including string corrections. In the case of a single gauge instanton configuration for the $\mathrm{SU}\left(N_{0}\right)$ factor, which corresponds to take $k_{0}=1$, this action is given by ${ }^{11}$

$$
\begin{aligned}
S_{\mathrm{D} 3 / \mathrm{D}(-1)}(\Phi, \bar{\Phi})= & \frac{2 \pi^{3} \alpha^{\prime 2}}{g_{s}} D_{c} D^{c}+\mathrm{i} D_{c}\left(\bar{w}_{\dot{\alpha}}\left(\tau^{c}\right)_{\dot{\beta}}^{\dot{\alpha}} w^{\dot{\beta}}\right)+\mathrm{i} \lambda_{\dot{\alpha}}\left(\bar{\mu} w^{\dot{\alpha}}+\bar{w}^{\dot{\alpha}} \mu\right) \\
+ & {\left[\frac{1}{2} \bar{w}_{\dot{\alpha}}(\Phi \bar{\Phi}+\bar{\Phi} \Phi) w^{\dot{\alpha}}+\frac{\mathrm{i}}{2} \bar{\mu}^{1} \bar{\Phi} \mu-\frac{\mathrm{i}}{2} \bar{\mu} \bar{\Phi} \mu^{1}\right.} \\
& \left.\quad+\mathrm{i} \bar{w}_{\dot{\alpha}}\left(\bar{D}^{\dot{\alpha}} \bar{\Phi}\right) \mu^{1}-\mathrm{i} \bar{\mu}^{1}\left(\bar{D}_{\dot{\alpha}} \bar{\Phi}\right) w^{\dot{\alpha}}\right]_{\bar{\theta}=0}
\end{aligned}
$$

In the first line above the quadratic term in the auxiliary fields $D_{c}$ with an $\alpha^{\prime}$-dependent coefficient comes from the gauge action $S_{G}$ in (2.12) written for a one-instanton configuration. The second line in (4.11) is the result of the replacements (4.5) and (4.7) in $S_{\phi}$, whereas the third line arises from (4.8) upon use of (4.10).

In the following we will discuss the non-perturbative effective terms that are induced on the D3 brane world volume by this instanton configuration.

\footnotetext{
${ }^{10}$ Couplings involving instanton moduli and chiral superfields of the form $\left.\bar{D}_{\dot{\alpha}} \bar{\Phi}\right|_{\bar{\theta}=0}$ have been recently considered in Refs. [54, 45, 57].

${ }^{11}$ Remember that in the one-instanton case for $\mathcal{N}=1$ models there are no $\chi$-moduli.
} 


\subsection{Field theory results: non-perturbative F-terms}

In the field theory limit $\alpha^{\prime} \rightarrow 0$, the instanton action (4.11) simplifies to

$$
\begin{aligned}
S_{\mathrm{D} 3 / \mathrm{D}(-1)}^{(0)}(\Phi, \bar{\Phi})= & \mathrm{i} D_{c}\left(\bar{w}_{\dot{\alpha}}\left(\tau^{c}\right)_{\dot{\beta}}^{\dot{\alpha}} w^{\dot{\beta}}\right)+\mathrm{i} \lambda_{\dot{\alpha}}\left(\bar{\mu} w^{\dot{\alpha}}+\bar{w}^{\dot{\alpha}} \mu\right) \\
+ & {\left[\frac{1}{2} \bar{w}_{\dot{\alpha}}(\Phi \bar{\Phi}+\bar{\Phi} \Phi) w^{\dot{\alpha}}+\frac{\mathrm{i}}{2} \bar{\mu}^{1} \bar{\Phi} \mu-\frac{\mathrm{i}}{2} \bar{\mu} \bar{\Phi} \mu^{1}\right.} \\
& \left.+\mathrm{i} \bar{w}_{\dot{\alpha}}\left(\bar{D}^{\dot{\alpha}} \bar{\Phi}\right) \mu^{1}-\mathrm{i} \bar{\mu}^{1}\left(\bar{D}_{\dot{\alpha}} \bar{\Phi}\right) w^{\dot{\alpha}}\right]_{\bar{\theta}=0} .
\end{aligned}
$$

Note that in this action $D_{c}$ and $\lambda_{\dot{\alpha}}$ appear only linearly and act as Lagrange multipliers for the bosonic and fermionic ADHM constraints, and that, as in (4.11), the dependence on the superspace coordinates $x^{\mu}$ and $\theta^{\alpha}$ is only through the matter superfields.

Integrating over all instanton moduli we obtain the following non-perturbative F-terms:

$$
S_{\text {n.p. }}=\int d^{4} x d^{2} \theta W_{\text {n.p. }}, \quad W_{\text {n.p. }}=\Lambda^{\beta_{0}} \int d \widehat{\mathfrak{M}} \mathrm{e}^{-S_{\mathrm{D} 3 / \mathrm{D}(-1)}^{(0)}(\Phi, \bar{\Phi})},
$$

where $\Lambda$ is the dynamically generated scale of the effective $\mathrm{SU}\left(N_{0}\right)$ SQCD theory we are considering, namely

$$
\Lambda^{\beta_{0}}=M_{s}^{\beta_{0}} \mathrm{e}^{2 \pi \mathrm{i} \tau_{0}} \quad \text { with } \quad \beta_{0}=3 N_{0}-N_{1}
$$

Despite the notation we have adopted, one should not immediately conclude that $W_{\text {n.p. }}$ defined in (4.13) be a superpotential since, as we will see momentarily, gauge instantons can induce also other types of non-perturbative F-terms.

In view of the explicit form of the field dependent moduli action (4.12), we can make the following general Ansatz:

$$
W_{\text {n.p. }}=\left.\mathcal{C} \Lambda^{\beta_{0}} \bar{\Phi}^{n} \Phi^{m}\left(\bar{D}_{\dot{\alpha}} \bar{\Phi} \bar{D}^{\dot{\alpha}} \bar{\Phi}\right)^{p}\right|_{\bar{\theta}=0},
$$

where $p$ is restricted to positive values to avoid the appearance of fermionic fields in the denominator. We now proceed as in Section 3.2 and require $W_{\text {n.p. }}$ to be a quantity of scaling dimension 3 and that its $\mathrm{U}(1)^{3}$ charges match those of the centered measure, given in (3.19) with $k_{1}=0$. Taking into account that $q[\bar{D} \bar{\Phi}]=+1 / 2$ and $q^{\prime}[\bar{D} \bar{\Phi}]=q^{\prime \prime}[\bar{D} \bar{\Phi}]=-1$, after some simple algebra we find that the parameters in (4.15) are given by

$$
p=-n=1-N_{0}+N_{1}, \quad m=1-N_{0}-N_{1} .
$$

The instanton induced effective interactions have thus the form

$$
W_{\text {n.p. }}=\left.\mathcal{C} \Lambda^{\beta_{0}} \frac{\left(\bar{D}_{\dot{\alpha}} \bar{\Phi} \bar{D}^{\dot{\alpha}} \bar{\Phi}\right)^{p}}{\bar{\Phi}^{p} \Phi^{p+2 N_{0}-2}}\right|_{\bar{\theta}=0}
$$

for $p=0,1, \ldots$

For $p=0$ (and hence for $N_{1}=N_{0}-1$ ) the above result reduces to the well-known ADS superpotential for $\mathrm{SU}\left(N_{c}\right) \mathrm{SQCD}$ with $N_{f}=N_{c}-1$ [49]; indeed, after using the D-flatness condition on the matter fields and explicitly performing the integrations over all ADHM 
moduli in this case, one can prove that the overall coefficient $\mathcal{C}$ is non-vanishing and that (4.17) becomes

$$
W_{\text {n.p. }}=\mathcal{C} \Lambda^{2 N_{c}+1} \frac{1}{\operatorname{det} M}
$$

where $M$ is the meson superfield, in agreement with the ADS result.

For $p>0$ the above result (4.17) reproduces the multi-fermion instanton induced interactions for SQCD with $N_{f} \geq N_{c}$ studied originally in [55] in the case $N_{c}=2$ and recently derived by integrating the ADHM moduli in [54] ${ }^{12}$. In particular, for $p=1$ and $N_{c}=N_{f}=2$, Eq. (4.17) yields the form

$$
W_{\text {n.p. }}=\left.\mathcal{C} \Lambda^{4} \frac{\bar{D}_{\dot{\alpha}} \bar{\Phi} \bar{D}^{\dot{\alpha}} \bar{\Phi}}{\bar{\Phi} \Phi^{3}}\right|_{\bar{\theta}=0}
$$

in accordance with the explicit result of the moduli integral $[55,54]$, which can be written as

$$
W_{\text {n.p. }}=\left.\mathcal{C} \Lambda^{4} \frac{\epsilon_{f_{1} f_{1}^{\prime}} \epsilon_{f_{2} f_{2}^{\prime}} \bar{D}_{\dot{\alpha}} \bar{M}_{f_{2}}^{f_{1}} \bar{D}^{\dot{\alpha}} \bar{M}_{f_{2}^{\prime}}^{f_{1}^{\prime}}+2 \bar{D}_{\dot{\alpha}} \bar{B} \bar{D}^{\dot{\alpha}} \overline{\tilde{B}}}{(\operatorname{tr} \bar{M} M+\bar{B} B+\overline{\tilde{B}} \tilde{B})^{3 / 2}}\right|_{\bar{\theta}=0},
$$

in terms of the $\mathrm{SU}(2)$ meson and baryon fields (see Eq.s (3.5) and (3.6) for $N_{f}=N_{c}=2$ ). For $p>1$ one obtains more general multi-fermion terms. As proved in Ref. [55], these multi-fermion terms, despite being non-holomorphic in the matter fields, are annihilated by the anti-chiral supercharges $\bar{Q}_{\dot{\alpha}}$, and as such they are genuine F-terms even if they do not correspond to a superpotential.

\section{Non-perturbative flux-induced effective interactions}

We now generalize the analysis of the previous sections and investigate the non-perturbative effects produced in the gauge theory by adding R-R and NS-NS fluxes in the internal space. In particular we consider the 3 -form flux

$$
G_{3}=F-\tau H
$$

which is made out of the R-R 3-form $F$, the NS-NS 3-form $H$, and the axion-dilaton $\tau$. The general couplings of closed string fluxes to open string fermionic bilinears were derived in [11] by evaluating mixed open/closed string amplitudes on disks with generalized mixed boundary conditions like those represented in Fig. 7. We refer to the above reference for a detailed discussion; here we simply recall that this world-sheet approach is particularly suited to study the case at hand and discuss how the $G_{3}$-fluxes couple to the instanton moduli ${ }^{13}$. Indeed, computing the mixed open/closed string diagrams of Fig. 7 in the

\footnotetext{
${ }^{12}$ See also Ref. [45] for related considerations in the case $N_{c}=1$.

${ }^{13}$ Notice that, differently from Ref. [11], here the transverse space to the D3-branes is non-compact. This implies that the normalization coefficients to be used here are like those computed in Ref. [11], but without the factors of the compactification volume $\mathcal{V}$.
} 

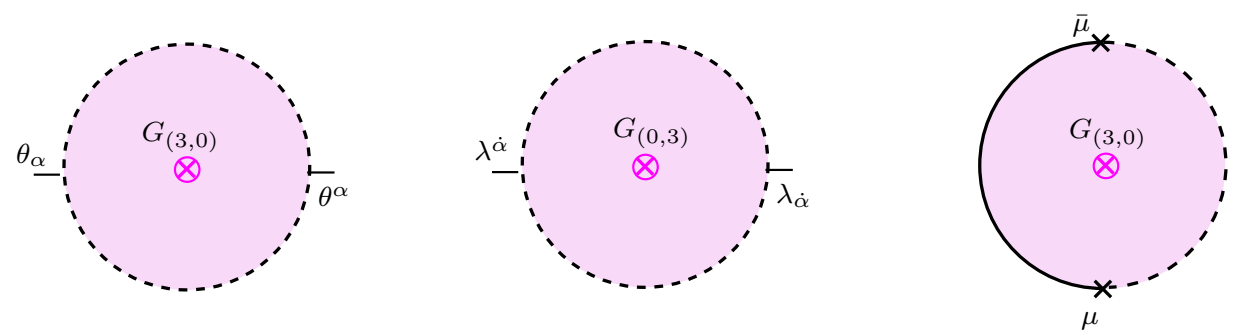

Figure 7: Diagrams encoding the linear couplings of bulk fluxes of type $(0,3)$ and $(3,0)$ to the instanton moduli reported in Eq. (5.2).

$\mathbb{Z}_{2} \times \mathbb{Z}_{2}$ orbifold, one finds that the flux induced interactions on the instanton moduli space are encoded in the action

$$
S^{\text {flux }}=2 \pi \mathrm{i}\left[\frac{2 G_{(3,0)}}{\sqrt{g_{s}}} \theta^{\alpha} \theta_{\alpha}-\frac{2 G_{(0,3)}}{\sqrt{g_{s}}} \frac{\pi^{2} \alpha^{\prime 2}}{2} \lambda_{\dot{\alpha}} \lambda^{\dot{\alpha}}\right]+\mathrm{i} \sqrt{g_{s}} G_{(3,0)} \bar{\mu} \mu,
$$

where we have denoted by $G_{(3,0)}$ and $G_{(0,3)}$ the $(3,0)$ and $(0,3)$ components of $G_{3}$ in the natural complex structure of the transverse space. These components satisfy, respectively, an imaginary self-duality and anti-self-duality condition and are responsible for the soft supersymmetry breaking terms related to the gravitino and gaugino masses, see e.g. [47, 48]. Note that in the first and last terms of (5.2) the scaling dimension of (length) ${ }^{-1}$ carried by the $G$-flux is compensated by the dimensions of $\theta, \mu$ and $\bar{\mu}$, while in the second term explicit $\alpha^{\prime}$ factors are needed. This is perfectly consistent with the fact that, while the $G_{(3,0)}$ flux components have a natural field theory interpretation as gaugino masses, the $G_{(0,3)}$ components instead have no counterpart on the gauge field theory. Thus, from the open string point of view their presence in (5.2) is a genuine string effect, as revealed also by the explicit factors of $\alpha^{\prime}$.

The action (5.2) can be conveniently rewritten as

$$
S^{\text {flux }}=\frac{2 \pi \mathrm{i}}{g_{s}}\left[G \theta^{\alpha} \theta_{\alpha}-\bar{G} \frac{\pi^{2} \alpha^{\prime 2}}{2} \lambda_{\dot{\alpha}} \lambda^{\dot{\alpha}}\right]+\frac{\mathrm{i}}{2} G \bar{\mu} \mu,
$$

where we have defined

$$
G=2 \sqrt{g_{s}} G_{(3,0)}, \quad \bar{G}=2 \sqrt{g_{s}} G_{(0,3)} .
$$

Eq. (5.3) is the form of the flux induced moduli action which we will use in the following to study the non-perturbative interactions generated by fractional D-instantons in the presence of bulk fluxes. In particular we will consider terms at the linear order in $G$ or $\bar{G}$ where the methods of [11] for the world-sheet derivation of the moduli action (5.3) are reliable. We therefore have two possibilities depending on whether we keep $G$ or $\bar{G}$ different from zero, which we are going to analyze in turn.

\subsection{One-instanton effects with $G \neq 0$}

In this case we can set $\bar{G}=0$ and look for the non-perturbative interactions proportional to $G$, assuming again that the fractional D-instanton is of type 0 , i.e. that $k_{0}=1$, as in 
Section 4. A class of such interactions is obtained by exploiting the $\frac{\mathrm{i}}{2} G \bar{\mu} \mu$ term of the flux action (5.3). At first order in $G$ this leads to

$$
S_{\text {n.p. }}(G)=\Lambda^{\beta_{0}} \int d^{4} x d^{2} \theta d \widehat{\mathfrak{M}} \mathrm{e}^{-S_{\mathrm{D} 3 / \mathrm{D}(-1)}(\Phi, \bar{\Phi})}\left(\frac{\mathrm{i}}{2} G \bar{\mu} \mu\right)
$$

where $S_{\mathrm{D} 3 / \mathrm{D}(-1)}(\Phi, \bar{\Phi})$ is the instanton action (4.11). By taking the limit $\alpha^{\prime} \rightarrow 0$ we obtain non-perturbative flux-induced terms in the effective action of the form

$$
S_{\text {n.p. }}(G)=\int d^{4} x d^{2} \theta W_{\text {n.p. }}(G), \quad W_{\text {n.p. }}(G)=\Lambda^{\beta_{0}} \int d \widehat{\mathfrak{M}} \mathrm{e}^{-S_{\mathrm{D} 3 / \mathrm{D}(-1)}^{(0)}(\Phi, \bar{\Phi})}\left(\frac{\mathrm{i}}{2} G \bar{\mu} \mu\right)
$$

where $S_{\mathrm{D} 3 / \mathrm{D}(-1)}^{(0)}(\Phi, \bar{\Phi})$ is the moduli action in the field theory limit given in (4.12). After performing the integration over all centered moduli, in the effective field theory we expect to find an interaction of the following schematic form

$$
W_{\text {n.p. }}(G)=\left.\mathcal{C} G \Lambda^{\beta_{0}} \bar{\Phi}^{n} \Phi^{m}\left(\bar{D}_{\dot{\alpha}} \bar{\Phi} \bar{D}^{\dot{\alpha}} \bar{\Phi}\right)^{p}\right|_{\bar{\theta}=0}
$$

with $\beta_{0}+n+m+3 p=2$ in order to have an operator of mass dimension 3 (remember that $G$ has dimensions of a mass). As before, we restrict to positive values of $p$ in order to avoid the appearance of fermionic fields in the denominator. Requiring that the three $\mathrm{U}(1)$ charges of $W_{\text {n.p. }}(G)$ match those of the centered instanton measure for consistency with (5.6), and using the information that $q(G)=-3$ and $q^{\prime}(G)=q^{\prime \prime}(G)=0$, it is easy to find that the parameters in (5.7) are given by

$$
p=-n-2=2-N_{0}+N_{1} \quad \text { and } \quad m=-N_{0}-N_{1} .
$$

The resulting multi-fermion interactions are non-supersymmetric as can be easily seen by noticing that they are non-holomorphic for $p=0$.

The case $p=1$ (i.e. $N_{1}=N_{0}-1$ ) is particularly interesting, since it corresponds to $\mathrm{SU}\left(N_{c}\right)$ SQCD with $N_{f}=N_{c}-1$. We have already recalled that in this case the gauge instanton induces the ADS superpotential; now we see that in the presence of a $G$-flux which softly breaks supersymmetry by giving a mass to the gaugino, the gauge instanton produces new types of low-energy effective interactions which are of the form

$$
W_{\text {n.p. }}(G)=\left.\mathcal{C} G \Lambda^{2 N_{c}+1} \frac{\bar{D}_{\dot{\alpha}} \bar{\Phi} \bar{D}^{\dot{\alpha}} \bar{\Phi}}{\bar{\Phi}^{3} \Phi^{2 N_{c}-1}}\right|_{\bar{\theta}=0} .
$$

We stress that this is a formal expression which only indicates the powers of the various fields that appear in the result; the precise structure of $W_{\text {n.p. }}(G)$ should be given in terms of the appropriate variables of the low-energy effective theory (the meson superfields in this case) and can be obtained by explicitly performing the integral over the instanton moduli which also dictates how color and flavor indices must be saturated. This task is particularly easy to do for SU(2) SQCD with one flavor, and some details can be found in Appendix A.3. There we show that for $N_{c}=2$ and $N_{f}=1$ the flux induced non-perturbative term (5.9) can be written as

$$
W_{\text {n.p. }}(G)=\left.\mathcal{C} G \Lambda^{5} \frac{\bar{D}^{2} \bar{M}}{(\bar{M} M)^{3 / 2}}\right|_{\bar{\theta}=0}
$$


where $M$ is the meson superfield of the effective theory. We can regard this interaction as a low-energy non-perturbative effect of the soft supersymmetry breaking realized by the $G$-flux in the microscopic high-energy theory. Finally, we observe that one can alternatively exploit the $G \theta^{2}$ term of the flux action (5.3) to produce non-supersymmetric interactions of the same type as the ones described here.

\subsection{One-instanton effects with $\bar{G} \neq 0$}

The contribution to the effective action linear in $\bar{G}$ in presence of a single fractional Dinstanton of type 0 is given, in analogy to Eq. (5.5), by

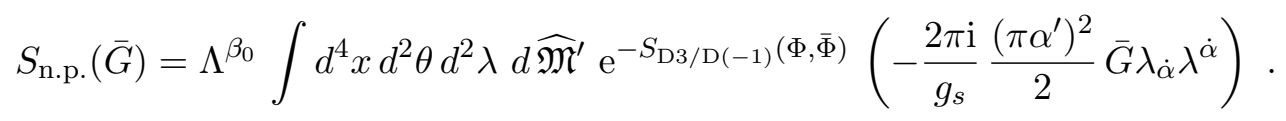

Here we have denoted by $\widehat{\mathfrak{M}}^{\prime}$ all centered moduli but $\lambda$. Performing the Grassmannian integration over $d^{2} \lambda$, we can write

$$
S_{\text {n.p. }}(\bar{G})=\int d^{4} x d^{2} \theta W_{\text {n.p. }}(\bar{G})
$$

where

$$
W_{\text {n.p. }}(\bar{G})=\left(\pi \alpha^{\prime}\right)^{2} \frac{2 \pi \mathrm{i}}{g_{s}} \Lambda^{\beta_{0}} \bar{G} \int d \widehat{\mathfrak{M}}^{\prime} \mathrm{e}^{-S_{\mathrm{D} 3 / \mathrm{D}(-1)}^{\prime}(\Phi, \bar{\Phi})}
$$

with $S_{\mathrm{D} 3 / \mathrm{D}(-1)}^{\prime}(\Phi, \bar{\Phi})$ being the action (4.11) without the fermionic ADHM constraint term since the Grassmannian integration over $\lambda$ has killed it. From (5.13) we therefore expect to find a result of the schematic form

$$
W_{\text {n.p. }}(\bar{G})=\left.\mathcal{C} \alpha^{\prime 2} \bar{G} \Lambda^{\beta_{0}} \bar{\Phi}^{n} \Phi^{m}\left(\bar{D}_{\dot{\alpha}} \bar{\Phi} \bar{D}^{\dot{\alpha}} \bar{\Phi}\right)^{p}\right|_{\bar{\theta}=0}+\cdots,
$$

where the dots stand for possible higher order string corrections. Requiring the equality of dimensions and $\mathrm{U}(1)^{3}$ charges between the definition (5.13) and the expression (5.14), one finds

$$
m=3-N_{0}-N_{1} \quad, \quad n=3+N_{0}-N_{1} \quad, \quad p=N_{1}-N_{0} .
$$

Let us focus on the simple case $p=0$ (i.e. $N_{0}=N_{1}$ ), which corresponds to a SQCD with $N_{f}=N_{c}$ flavors. In this case, in absence of fluxes, one gets only multi-fermion terms of Beasley-Witten type, like the ones displayed in Eq.s (4.19) and (4.20). In the presence of a $\bar{G}$-flux we have also a non-holomorphic contribution of the form

$$
W_{\text {n.p. }}=\left.\mathcal{C} \alpha^{\prime 2} \bar{G} \Lambda^{2 N_{c}} \bar{\Phi}^{3} \Phi^{3-2 N_{c}}\right|_{\bar{\theta}=0}
$$

which can be explicitly computed by performing the integration over $d \widehat{\mathfrak{M}^{\prime}}$, as shown in Appendix A.4. Notice that again these terms are non-holomorphic and therefore manifestly non-supersymmetric. For $N_{c}=2$, the result is

$$
W_{\text {n.p. }}=\left.\mathcal{C} \alpha^{\prime 2} \bar{G} \Lambda^{4} \frac{\operatorname{det} \bar{M}}{(\operatorname{tr} \bar{M} M+\bar{B} B+\overline{\tilde{B}} \tilde{B})^{1 / 2}}\right|_{\bar{\theta}=0},
$$

where $M$ is the meson superfield and $B$ and $\widetilde{B}$ are the baryon superfields. 


\section{Stringy instanton effects in presence of fluxes}

D-instantons of type 2 and 3 are of different type with respect to the D3 branes where the SQCD-like $\mathrm{SU}\left(N_{0}\right) \times \mathrm{SU}\left(N_{1}\right)$ theory is defined, and lead thus to "stringy" or "exotic" non-perturbative effects. In this case we have only fermionic mixed moduli $\mu^{2}, \bar{\mu}^{2}$ and $\mu^{3}$, $\bar{\mu}^{3}$, while there are no $w_{\dot{\alpha}}$ and $\bar{w}_{\dot{\alpha}}$ 's from the NS sectors.

Let us now derive the general form of the centered partition function in dependence of the vacuum expectation value of the scalar $\phi$, in analogy to what we did for the gauge instantons in Section 3. The moduli action (2.11) drastically simplifies. In particular, it is holomorphic in $\phi$ and does not contain any $\lambda$ dependence. Therefore, unless one introduces an orientifold projection $[28,29,30]$ or invokes other mechanisms $[35,39,45]$, the only way to get a non-zero result is to include the flux-induced $\bar{G} \lambda \lambda$ term of Eq. (5.2) and use it to perform the $\lambda$ integration. At the linear level in the fluxes, the other flux interactions in (5.3) become then irrelevant. Neglecting as usual numerical prefactors, we can write the centered partition function for a stringy instanton configuration with instanton numbers $k_{2}$ and $k_{3}$ as

$$
W_{\text {n.p. }}(\bar{G})=\alpha^{\prime 2} \bar{G} M_{s}^{k_{2} \beta_{2}+k_{3} \beta_{3}} \mathrm{e}^{2 \pi \mathrm{i}\left(k_{2} \tau_{2}+k_{3} \tau_{3}\right)} \int d \widehat{\mathfrak{M}}^{\prime} \mathrm{e}^{-S_{\mathrm{D} 3 / \mathrm{D}(-1)}},
$$

leading to the following general Ansatz

$$
W_{\text {n.p. }}(\bar{G})=\mathcal{C} \bar{G} M_{s}^{k_{2} \beta_{2}+k_{3} \beta_{3}+n} \mathrm{e}^{2 \pi \mathrm{i}\left(k_{2} \tau_{2}+k_{3} \tau_{3}\right)} \phi^{m} .
$$

We have not fixed a priori the power of $M_{s} \sim 1 / \sqrt{\alpha^{\prime}}$ since in this case the moduli integration can produce extra factors of $\alpha^{\prime}$ with respect to those appearing in Eq. (6.1), because of the $S_{G}$ part of the moduli action $(2.11)$ which appears with an explicit $\left(\alpha^{\prime}\right)^{2}$ in front ${ }^{14}$.

The equality between the $q, q^{\prime}$ and $q^{\prime \prime}$ charges following from this ansatz and those implied by the definition (6.1) plus the request that the mass dimension of $W_{\text {n.p. }}$. be equal to 3 impose that

$$
n=2 \quad, \quad m=2\left(k_{2} N_{0}+k_{3} N_{1}\right),
$$

and

$$
\left(k_{2}-k_{3}\right)\left(N_{0}-N_{1}\right)=0 ;
$$

To derive these equations we have used the fact that for our brane configuration $\beta_{2}=\beta_{3}=$ $-\left(N_{0}+N_{1}\right)$. The condition (6.4) admits the following solutions:

$$
\begin{gathered}
k_{2}=k_{3} \quad \text { with } \quad N_{0} \text { and } N_{1} \text { arbitrary } \\
N_{0}=N_{1} \quad \text { with } \quad k_{2} \text { and } k_{3} \text { arbitrary } .
\end{gathered}
$$

The centered partition function in stringy instanton sectors can thus be written in the form

$$
W_{\text {n.p. }}(\bar{G})=\mathcal{C} \bar{G} M_{s}^{k_{2} \beta_{2}+k_{3} \beta_{3}+2} \mathrm{e}^{2 \pi \mathrm{i}\left(k_{2} \tau_{2}+k_{3} \tau_{3}\right)} \phi^{-\left(k_{2} \beta_{2}+k_{3} \beta_{3}\right)} .
$$

\footnotetext{
${ }^{14}$ In particular, the "center of mass" part of the $D_{c}$ 's appears only through the quadratic term $\sim \alpha^{\prime 2} D_{c} D^{c}$ and the gaussian integration over it produces negative powers of $\sqrt{\alpha^{\prime}}$. This is different with respect to the gauge instanton cases considered in Sections 4 and 5 , where the $D_{c}$ 's couple also to the bosonic moduli $w$ and $\bar{w}$, leading to a completely different type of integral.
} 
Let us now concentrate on the set-up containing a single stringy instanton described in Fig. $3 b$ ), namely let us set $k_{2}=1, k_{3}=0$. In this case it is easy to promote the vacuum expectation value $\phi$ to the full superfield $\Phi(x, \theta)$ through diagrams such as those of Fig. 4. and the moduli integration can be explicitly done. As remarked above, the only way to saturate the Grassmannian integration over $d \lambda_{\dot{\alpha}}$ is via their $\bar{G}$ interaction and the non-perturbative contribution to the effective action of this "stringy" instanton sector is

$$
S_{\text {n.p. }}=\int d^{4} x d^{2} \theta W_{\text {n.p. }}(\bar{G})
$$

where the superpotential is given by

$$
W_{\text {n.p. }}=\mathcal{C} \alpha^{\prime 2} M_{s}^{-\left(N_{0}+N_{1}\right)} \mathrm{e}^{2 \pi \mathrm{i} \tau_{2}} \bar{G} \int d \widehat{\mathfrak{M}}^{\prime} \mathrm{e}^{-S_{\mathrm{D} 3 / \mathrm{D}(-1)}(\Phi)}
$$

Notice that the dimensional prefactor does not combine with the exponential of the classical action to form the dynamically generated scale of the gauge theory, since $\tau_{2}$ is the complexified coupling of D3-branes of type 2, which are not the ones that support the gauge theory we are considering.

The moduli $\widehat{\mathfrak{M}^{\prime}}$ appearing in (6.8) are simply $\left\{D_{c}, \mu^{2} \bar{\mu}^{2}, \mu^{3} \bar{\mu}^{3}\right\}$, with $\mu^{2}$ and $\mu^{3}$ transforming in the fundamental representations of $\mathrm{U}\left(N_{0}\right)$ and $\mathrm{U}\left(N_{1}\right)$ respectively. Thus, the moduli action to be used in (6.8) simply reduces to

$$
S_{\mathrm{D} 3 / \mathrm{D}(-1)}(\Phi)=\frac{2 \pi^{3} \alpha^{\prime 2}}{g_{s}} D_{c} D^{c}-\frac{\mathrm{i}}{2}\left(\bar{\mu}^{3} \Phi \mu^{2}-\bar{\mu}^{2} \Phi \mu^{3}\right) .
$$

Hence, the integral in (6.8) explicitly reads

$$
\int d^{3} D d^{N_{0}} \mu^{2} d^{N_{0}} \bar{\mu}^{2} d^{N_{1}} \mu^{3} d^{N_{1}} \bar{\mu}^{3} \mathrm{e}^{-\frac{2 \pi^{3} \alpha^{\prime 2}}{g_{s}} D_{c} D^{c}+\frac{i}{2}\left(\bar{\mu}^{3} \Phi \mu^{2}-\bar{\mu}^{2} \Phi \mu^{3}\right)} .
$$

The integration over the $\mu$ 's clearly vanishes unless $N_{0}=N_{1}$, in which case we get, after performing also the gaussian integration over the $D$ 's,

$$
\alpha^{\prime-3} \operatorname{det} Q \operatorname{det} \tilde{Q}=\alpha^{\prime-3} \operatorname{det} M
$$

Here we have used the form (3.3) of $\Phi$ and in the last step we have introduced the meson field $M=\tilde{Q} Q$. We have also disregarded all numerical constants and kept track only of the powers of $\alpha^{\prime} \propto M_{s}^{-2}$, since in all of our treatment we have specified completely only the dimensional part of the prefactors in the moduli measure.

Inserting (6.11) into (6.8) we find therefore that a single stringy instanton in presence of an imaginary self-dual three-form flux produces for $N_{0}=N_{1}$ (i.e. for a SQCD with $N_{f}=N_{c}$ flavors) a holomorphic superpotential

$$
W_{\text {n.p. }}=\mathcal{C} M_{s}^{2-2 N_{c}} \mathrm{e}^{2 \pi \mathrm{i} \tau_{2}} \bar{G} \operatorname{det} M
$$

Interestingly, the interactions generated by stringy instantons are still holomorphic and therefore supersymmetric even in the presence of the supersymmetry breaking flux $\bar{G}$. it 
would be interesting to investigate the implications of such non-perturbative terms for the low-energy effective action.

\section{Acknowledgments}

We thank B. Acharya, C. Angelantonj, C. Bachas, K.S. Narain, I. Pesando, R. Russo and T. Weigand for useful discussions. We furthermore thank the referee for questions and clarifying comments. This work is partially supported by the European Commission FP6 Programme under contract MRTN-CT-2004-005104 "Constituents, Fundamental Forces and Symmetries of the Universe", in which A.L. is associated to University of Torino, MRTN-CT-2004-512194 "Superstring Theory" and MRTN-CT-2004-503369 "The Quest for Unification: Theory Confronts Experiment", by the Italian MIUR-PRIN contract 20075ATT78 and by the NATO grant PST.CLG.978785. L.F. would like to thank C. Bachas for kind support and LPTENS for wonderful hospitality.

\section{A. Appendix}

\section{A.1 Gauge coupling and instanton action}

Let us consider a $\mathrm{D} p$-brane wrapping a $(p-3)$-cycle $\mathcal{C}_{A}$ and denote by $\tau_{A}$ the complexified gauge coupling of the resulting four-dimensional super Yang-Mills theory:

$$
\tau_{A}=\frac{\theta_{A}}{2 \pi}+\mathrm{i} \frac{4 \pi}{g_{A}^{2}}
$$

A gauge instanton in this theory can be described in terms of a Euclidean $(p-4)$-brane wrapping the same $(p-3)$-cycle $\mathcal{C}_{A}$. The instanton induces non-perturbative interactions weighted by $\mathrm{e}^{-k_{A} S_{A}^{\mathrm{E}(p-4)}}$ with $k_{A}$ being the number of instantonic branes and $S_{A}$ the action for a single instanton. Aim of this appendix is to show the relation

$$
S_{A}^{\mathrm{E}(p-4)}=-2 \pi \mathrm{i} \tau_{A} .
$$

which justifies the form of $W_{\text {n.p. }}$ in (1.4).

Eq. (A.2) follows from a comparison of the world-volume action of the Euclidean $\mathrm{E}(p-4)$-brane with that of the wrapped D $p$-brane [21]. In Euclidean signature, the latter is ${ }^{15}$

$$
S_{A}^{\mathrm{D} p}=\mu_{p} \operatorname{Tr}\left[\int_{\mathbb{R}^{4} \times \mathcal{C}_{A}} \mathrm{e}^{-\varphi} \sqrt{\operatorname{det}\left(g+2 \pi \alpha^{\prime} F\right)}-\mathrm{i} \int_{\mathbb{R}^{4} \times \mathcal{C}_{A}} \sum_{n} C_{2 n} \mathrm{e}^{2 \pi \alpha^{\prime} F}\right],
$$

where $\mu_{p}=(2 \pi)^{-p}\left(\alpha^{\prime}\right)^{-(p+1) / 2}$ is the D $p$-brane tension, $\varphi$ the dilaton, $g$ the string frame metric and $C_{2 n}$ the R-R $2 n$-form potentials. Expanding (A.3) to quadratic order in $F$ and

\footnotetext{
${ }^{15}$ Here we assume $\left[F_{\mu \nu}, F_{\sigma \rho}\right]=0$ and take $F=F_{i} T^{j}$ with $\operatorname{Tr}\left(T^{i} T^{j}\right)=\frac{1}{2} \delta^{i j}$ and $i, j$ running in the adjoint of the gauge.
} 
comparing with the standard form of the Yang-Mills action in Euclidean signature, we find that the complexified four-dimensional gauge coupling is

$$
\tau_{A}=2 \pi\left(2 \pi \alpha^{\prime}\right)^{2} \mu_{p} \int_{\mathcal{C}_{A}}\left[C_{p-3}+\mathrm{ie}^{-\varphi} \sqrt{\operatorname{det} g}\right] .
$$

On the other hand the action for a Euclidean $(p-4)$-brane wrapping $\mathcal{C}_{A}$ is given by

$$
S_{A}^{\mathrm{E}(p-4)}=\mu_{p-4}\left[\int_{\mathcal{C}_{A}} \mathrm{e}^{-\varphi} \sqrt{\operatorname{det} g}-\mathrm{i} \int_{\mathcal{C}_{A}} C_{p-3}\right]=-2 \pi \mathrm{i} \tau_{A}
$$

in agreement with (A.2).

\section{A.2 Vertex operators for gauge fields and instanton moduli}

In this subsection we list the vertex operators of the various fields and moduli of our model, including their normalizations which we express in terms of the unit of length $\left(2 \pi \alpha^{\prime}\right)^{\frac{1}{2}}$. In our conventions the vertex operators are always dimensionless and, in general, we assign canonical dimensions to their polarizations, namely dimensions of (length) ${ }^{-1}$ to bosonic fields and dimensions of (length) $)^{-3 / 2}$ to fermionic ones. However, in the instanton sector, some of the ADHM moduli acquire different dimensions as indicated in Tab. 3. The vertex operators we list in the following are written using the open string fields appropriate for the $\mathrm{D} 3 / \mathrm{D}(-1)$ system, namely the space-time bosonic and fermionic string coordinates $X^{\mu}$ and $\psi^{\mu}$, the transverse bosonic and fermionic string coordinates in the complex basis $Z^{I}$ and $\Psi^{I}$, the space-time spin-fields $S_{\alpha}$ and $S^{\dot{\alpha}}$, and the internal ones $S_{A}$ and $S^{A}$. Moreover we denote by $\phi$ the bosonic field of the superghost system. For more details we refer to $[18,22]$.

Vertices for gauge fields The gauge fields originate from D3/D3 strings. They include a gauge vector $A_{\mu}$ and a gaugino $\Lambda^{\alpha}$ with its conjugate $\bar{\Lambda}_{\dot{\alpha}}$ transforming in the adjoint representation, plus bi-fundamental matter fields. The corresponding vertices at momentum $p$ are

$$
\begin{aligned}
V_{A} & =\left(\pi \alpha^{\prime}\right)^{\frac{1}{2}}\left(A_{\mu}\right)^{u_{A}} v_{v_{A}} \psi^{\mu} \mathrm{e}^{-\phi} \mathrm{e}^{\mathrm{i} p \cdot X}, \\
V_{\Lambda} & =\left(2 \pi \alpha^{\prime}\right)^{\frac{3}{4}}\left(\Lambda^{\alpha}\right)^{u_{A}} S_{v_{A}} S_{0} \mathrm{e}^{-\phi / 2} \mathrm{e}^{\mathrm{i} p \cdot X}, \\
V_{\bar{\Lambda}} & =\left(2 \pi \alpha^{\prime}\right)^{\frac{3}{4}}\left(\bar{\Lambda}_{\dot{\alpha}}\right)^{u_{A}}{ }_{v_{A}} S^{\dot{\alpha}} S^{0} \mathrm{e}^{-\phi / 2} \mathrm{e}^{\mathrm{i} p \cdot X},
\end{aligned}
$$

for the adjoint fields, and

$$
\begin{aligned}
V_{\phi^{I}} & =\left(\pi \alpha^{\prime}\right)^{\frac{1}{2}}\left(\phi^{I}\right)_{v_{A \otimes I}^{u_{A}}} \bar{\Psi}_{I} \mathrm{e}^{-\phi} \mathrm{e}^{\mathrm{i} p \cdot X}, \\
V_{\bar{\phi}_{I}} & =\left(\pi \alpha^{\prime}\right)^{\frac{1}{2}}\left(\bar{\phi}_{I}\right)^{u_{A \otimes I}} \Psi^{I} \mathrm{e}^{-\phi} \mathrm{e}^{\mathrm{i} p \cdot X}, \\
V_{\psi^{I}} & =\left(2 \pi \alpha^{\prime}\right)^{\frac{3}{4}}\left(\psi^{\alpha I}\right)^{u_{A}} S_{v_{A \otimes I}} S_{I} \mathrm{e}^{-\phi / 2} \mathrm{e}^{\mathrm{i} p \cdot X}, \\
V_{\bar{\psi}_{I}} & =\left(2 \pi \alpha^{\prime}\right)^{\frac{3}{4}}\left(\bar{\psi}_{\dot{\alpha} I}\right)^{u_{A \otimes I}} S^{\dot{\alpha}} S^{I} \mathrm{e}^{-\phi / 2} \mathrm{e}^{\mathrm{i} p \cdot X}, \\
V_{F^{I}} & =\pi \alpha^{\prime}\left(F^{I}\right)_{v_{A \otimes I}^{u_{A}}}^{\epsilon_{I J K}} \Psi^{J} \Psi^{K} \mathrm{e}^{\mathrm{i} p \cdot X}, \\
V_{\bar{F}_{I}} & =\pi \alpha^{\prime}\left(\bar{F}_{I}\right)_{v_{A \otimes I}^{u_{A}}}^{I J K} \bar{\Psi}_{J} \bar{\Psi}_{K} \mathrm{e}^{\mathrm{i} p \cdot X},
\end{aligned}
$$


for the bi-fundamental matter fields. The internal spin fields appearing in the above formulas are

$$
\begin{aligned}
& S^{0}=S^{+++}, S^{1}=S^{+--}, S^{2}=S^{-+-}, S^{3}=S^{-++} \\
& S_{0}=S_{---}, S_{1}=S_{-++}, S_{2}=S_{+-+}, S_{3}=S_{++-},
\end{aligned}
$$

where the \pm 's denote the signs of the spinorial weights of $\mathrm{SO}(6)$. Notice that $S^{A}$ and $S_{A}$ trasform in the representation $R_{A}$ of the orbifold group.

Vertices for instanton moduli Strings with at least one end-point on a $D(-1)$ brane give rise to moduli rather than dynamical fields because either they do not have longitudinal Neumann directions at all or they have mixed boundary conditions. The vertex operators for $\mathrm{D}(-1) / \mathrm{D}(-1)$ moduli with alike end-points are

$$
\begin{aligned}
V_{a} & =\sqrt{2} g_{0}\left(\pi \alpha^{\prime}\right)^{\frac{1}{2}}\left(a_{\mu}\right)_{j_{A}}^{i_{A}} \psi^{\mu} \mathrm{e}^{-\phi}, \\
V_{D} & =2 \pi \alpha^{\prime}\left(D_{c}\right)_{j_{A}}^{i_{A}} \bar{\eta}_{\mu \nu}^{c} \psi^{\mu} \psi^{\nu}, \\
V_{M} & =\frac{g_{0}}{\sqrt{2}}\left(2 \pi \alpha^{\prime}\right)^{\frac{3}{4}}\left(M_{\alpha}\right)_{j_{A}}^{i_{A}} S_{\alpha} S_{0} \mathrm{e}^{-\phi / 2}, \\
V_{\lambda} & =\left(2 \pi \alpha^{\prime}\right)^{\frac{3}{4}}\left(\lambda_{\dot{\alpha}}\right)_{j_{A}}^{i_{A}} S^{\dot{\alpha}} S^{0} \mathrm{e}^{-\phi / 2},
\end{aligned}
$$

where D-instanton gauge coupling $g_{0}$ is expressed in terms of $\alpha^{\prime}$ and $g_{s}$ as in (2.15). If the end-points are different we instead have

$$
\begin{aligned}
V_{\chi^{I}} & =\left(\pi \alpha^{\prime}\right)^{\frac{1}{2}}\left(\chi^{I}\right)_{j_{A \otimes I}}^{i_{A}} \bar{\Psi}_{I} \mathrm{e}^{-\phi}, \\
V_{\bar{\chi}_{I}} & =\left(\pi \alpha^{\prime}\right)^{\frac{1}{2}}\left(\bar{\chi}_{I}\right)_{j_{A} i_{A}}^{i^{I}} \Psi^{I} \mathrm{e}^{-\phi}, \\
V_{M^{I}} & =\frac{g_{0}}{\sqrt{2}}\left(2 \pi \alpha^{\prime}\right)^{\frac{3}{4}}\left(M^{\alpha I}\right)_{j_{A \otimes I}}^{i_{A}} S_{\alpha} S_{I} \mathrm{e}^{-\phi / 2}, \\
V_{\lambda_{I}} & =\left(2 \pi \alpha^{\prime}\right)^{\frac{3}{4}}\left(\lambda_{\dot{\alpha} I}\right)_{j_{A}}^{i_{A \otimes I}} S^{\dot{\alpha}} S^{I} \mathrm{e}^{-\phi / 2} .
\end{aligned}
$$

The moduli of the charged sector arise from open strings with $\mathrm{D} 3 / \mathrm{D}(-1)$ or $\mathrm{D}(-1) / \mathrm{D} 3$ boundary conditions. The vertex operators for those which in the field theory limit describe gauge instantons are

$$
\begin{aligned}
V_{w} & =\frac{g_{0}}{\sqrt{2}}\left(2 \pi \alpha^{\prime}\right)^{\frac{1}{2}}\left(w_{\dot{\alpha}}\right)_{j_{A}}^{u_{A}} \Delta S^{\dot{\alpha}} \mathrm{e}^{-\phi}, \\
V_{\bar{w}} & =\frac{g_{0}}{\sqrt{2}}\left(2 \pi \alpha^{\prime}\right)^{\frac{1}{2}}\left(\bar{w}_{\dot{\alpha}}\right)_{u_{A}}^{i_{A}} \bar{\Delta} S^{\dot{\alpha}} \mathrm{e}^{-\phi}, \\
V_{\mu} & =\frac{g_{0}}{\sqrt{2}}\left(2 \pi \alpha^{\prime}\right)^{\frac{3}{4}}(\mu)_{j_{A}}^{u_{A}} \Delta S_{0} \mathrm{e}^{-\phi / 2}, \\
V_{\bar{\mu}} & =\frac{g_{0}}{\sqrt{2}}\left(2 \pi \alpha^{\prime}\right)^{\frac{3}{4}}(\mu)_{u_{A}}^{i_{A}} \bar{\Delta} S_{0} \mathrm{e}^{-\phi / 2},
\end{aligned}
$$

while those related to stringy instantons are

$$
\begin{aligned}
V_{\mu^{I}} & =\frac{g_{0}}{\sqrt{2}}\left(2 \pi \alpha^{\prime}\right)^{\frac{3}{4}}\left(\mu^{I}\right)_{i_{A \otimes I}}^{u_{A}} \Delta S_{I} \mathrm{e}^{-\phi / 2}, \\
V_{\bar{\mu}_{I}} & =\frac{g_{0}}{\sqrt{2}}\left(2 \pi \alpha^{\prime}\right)^{\frac{3}{4}}\left(\bar{\mu}_{I}\right)_{{ }_{u_{A}}^{i_{A \otimes I}}}^{i_{\Delta}} S_{I} \mathrm{e}^{-\phi / 2},
\end{aligned}
$$

where $\Delta$ and $\bar{\Delta}$ are the bosonic twist and anti-twist operators respectively and encode the change of boundary condition from Neumann to Dirichlet and vice-versa. 


\section{A.3 Derivation of the non-perturbative flux effects for $N_{f}=N_{c}-1$}

As discussed in Section 5.1, the non-perturbative interactions induced by the flux $G$ are obtained at linear order by computing the integral (see Eq. (5.6))

$$
W_{\text {n.p. }}(G)=\Lambda^{\beta_{0}} \int d \widehat{\mathfrak{M}} \mathrm{e}^{-S_{\mathrm{D} 3 / \mathrm{D}(-1)}^{(0)}(\Phi, \bar{\Phi})}\left(\frac{\mathrm{i}}{2} G \bar{\mu} \mu\right)
$$

where the action on the moduli space is given in Eq. (4.12). As we have seen we expect a non-vanishing contribution to this integral when $N_{f}=N_{c}-1$, whose schematic form is given in Eq. (5.9).

Here we consider in detail the case of the SU(2) theory, i.e. $N_{c}=2$ and $N_{f}=1$. Denoting by $Q^{u}$ and $\widetilde{Q}_{u}$ (with $u=1,2$ ) the fundamental and anti-fundamental blocks of the matter superfield $\Phi$ (see Eq. (3.3)), and by $\mu^{\prime}$ and $\bar{\mu}^{\prime}$ the non-trivial components of $\mu^{1}$ and $\bar{\mu}^{1}$ respectively, the moduli action (4.12) in this case becomes

$$
\begin{aligned}
S_{\mathrm{D} 3 / \mathrm{D}(-1)}^{(0)}= & \mathrm{i} D_{c}\left(\bar{w}_{\dot{\alpha} u}\left(\tau^{c}\right)_{\dot{\beta}}^{\dot{\alpha}} w^{\dot{\beta} u}\right)+\mathrm{i} \lambda_{\dot{\alpha}}\left(\bar{\mu}_{u} w^{\dot{\alpha} u}+\bar{w}_{u}^{\dot{\alpha}} \mu^{u}\right) \\
+ & {\left[\frac{1}{2} \bar{w}_{\dot{\alpha} u}\left(Q^{u} \bar{Q}_{v}+\overline{\widetilde{Q}}^{u} \widetilde{Q}_{v}\right) w^{\dot{\alpha} v}+\frac{\mathrm{i}}{2} \bar{\mu}^{\prime} \bar{Q}_{u} \mu^{u}-\frac{\mathrm{i}}{2} \bar{\mu}_{u} \overline{\widetilde{Q}}^{u} \mu^{\prime}\right.} \\
& \left.+\mathrm{i} \bar{w}_{\dot{\alpha} u}\left(\bar{D}^{\dot{\alpha}} \overline{\widetilde{Q}}^{u}\right) \mu^{\prime}-\mathrm{i} \bar{\mu}^{\prime}\left(\bar{D}_{\dot{\alpha}} \bar{Q}_{u}\right) w^{\dot{\alpha} u}\right]_{\bar{\theta}=0}
\end{aligned}
$$

where we have explicitly indicated also the two-valued color indices.

The integration over $d^{2} \lambda$ allows to soak up the two fermionic zero-modes $\mu$ and $\bar{\mu}$ left after the $G$-flux insertion. After some elementary algebra, one finds a contribution simply proportional to

$$
\bar{w}_{\dot{\alpha} u} w^{\dot{\alpha} u} .
$$

To saturate the Grassmannian integrals over $d \mu^{\prime}$ and $d \bar{\mu}^{\prime}$ the only option is to bring down the terms containing $\bar{D}_{\dot{\alpha}} \overline{\widetilde{Q}}$ and $\bar{D}_{\dot{\alpha}} \bar{Q}$ from the moduli action, thus obtaining a contribution proportional to

$$
\left.\left(\bar{D}^{\dot{\alpha}} \overline{\widetilde{Q}}^{u} \bar{D}^{\dot{\beta}} \bar{Q}_{v}\right)\right|_{\bar{\theta}=0} \bar{w}_{\dot{\alpha} u} w_{\dot{\beta}}^{v} .
$$

Thus, after integrating over all fermionic instanton moduli, we are left with

$$
W_{\text {n.p. }}(G)=\mathcal{C} G \Lambda^{5}\left(\bar{D}^{\dot{\alpha}} \overline{\widetilde{Q}}^{u} \bar{D}^{\dot{\beta}} \bar{Q}_{v}\right) \int d^{4} w d^{4} \bar{w} d^{3} D \mathrm{e}^{-\mathrm{i} D_{c} \bar{w} \tau^{c} w-\frac{1}{2} \bar{w}(Q \bar{Q}+\overline{\widetilde{Q}} \widetilde{Q}) w} \bar{w}_{\dot{\alpha} u} w_{\dot{\beta}}^{v} \bar{w}_{\dot{\gamma} r} w^{\dot{\gamma} r}
$$

where we have clumped all numerical constants in the normalization factor $\mathcal{C}$ and understood that we must set $\bar{\theta}=0$ in the right hand side. The bosonic integral (A.17) has been evaluated in Ref. [54] (see in particular Eq. (5.7) of the published version) and the result is

$$
\int d^{4} w d^{4} \bar{w} d^{3} D \mathrm{e}^{-\mathrm{i} D_{c} \bar{w} \tau^{c} w-\bar{w} A w} \bar{w}_{\dot{\alpha} u} w_{\dot{\beta}}^{v} \bar{w}_{\dot{\gamma} r} w^{\dot{\gamma} r}=\frac{2 \epsilon_{\dot{\alpha} \dot{\beta}} \delta_{u}^{v}}{(\operatorname{tr} A)^{3}}
$$

where $A$ is the $2 \times 2$ hermitian matrix $A=\frac{1}{2}(Q \bar{Q}+\overline{\widetilde{Q}} \widetilde{Q})$. Exploiting the D-flatness condition (3.4) for $\xi=0$, it is easy to prove that

$$
\operatorname{tr} A=(\bar{M} M)^{1 / 2}
$$


where $M=\widetilde{Q}_{u} Q^{u}$ is the meson superfield. Using these results in (A.17), after some simple manipulations and absorbing all numerical factors by redefining the overall coefficient $\mathcal{C}$, we finally obtain

$$
W_{\text {n.p. }}(G)=\left.G \Lambda^{5} \frac{\bar{D}^{2} \bar{M}}{(\bar{M} M)^{3 / 2}}\right|_{\bar{\theta}=0} .
$$

As mentioned in the main text, this explicit result is in full agreement with the general expression (5.9) obtained using dimensional analysis and $\mathrm{U}(1)^{3}$ charge conservation.

\section{A.4 Derivation of the non-perturbative flux effects for $N_{f}=N_{c}$}

Let us now consider the effective interaction induced by a $\bar{G}$ background flux which, according to Eq. (5.13), is given by

$$
W_{\text {n.p. }}(\bar{G})=\left(\pi \alpha^{\prime}\right)^{2} \frac{2 \pi \mathrm{i}}{g_{s}} \Lambda^{\beta_{0}} \bar{G} \int d \widehat{\mathfrak{M}}^{\prime} \mathrm{e}^{-S_{\mathrm{D} 3 / \mathrm{D}(-1)}(\Phi, \bar{\Phi})}
$$

where in the moduli action it is understood that we have to set $\bar{\theta}=0$.

We focus on the case $N_{0}=N_{1}$, corresponding to SQCD with $N_{c}=N_{f}$ flavors. The moduli action to be used in (A.21) reads explicitly

$$
\begin{aligned}
\left.S_{\mathrm{D} 3 / \mathrm{D}(-1)}\right|_{\bar{\theta}=0}= & \mathrm{i} D_{c}\left(\bar{w}_{\dot{\alpha} u}\left(\tau^{c}\right)_{\dot{\beta}}^{\dot{\alpha}} w^{\dot{\beta} u}\right)+\left[\frac{1}{2} \bar{w}_{\dot{\alpha} u}\left(Q^{u}{ }_{f} \bar{Q}_{v}^{f}+\overline{\widetilde{Q}}_{f}^{u} \widetilde{Q}_{v}^{f}\right) w^{\dot{\alpha} v}\right. \\
& \left.+\frac{\mathrm{i}}{2} \bar{\mu}_{f}^{1} \bar{Q}_{u}^{f} \mu^{u}-\frac{\mathrm{i}}{2} \bar{\mu}_{u} \overline{\widetilde{Q}}_{f}^{u} \mu^{1 f}+\mathrm{i} \bar{w}_{\dot{\alpha} u}\left(\bar{D}^{\dot{\alpha}} \overline{\widetilde{Q}}\right)_{f}^{u} \mu^{1 f}-\mathrm{i} \bar{\mu}_{f}^{1}\left(\bar{D}_{\dot{\alpha}} \bar{Q}\right)^{f}{ }_{u} w^{\dot{\alpha} u}\right]_{\bar{\theta}=0}
\end{aligned}
$$

where $u$ and $f$ are fundamental color and flavor indices respectively. With respect to Eq. (4.11) we have neglected the quadratic term in $D_{c}$ 's since it appears with an explicit $\alpha^{\prime 2}$ in front, and it leads to effects of higher order in $\alpha^{\prime}$ with respect to the ones we will compute in the following.

Since we have chosen $N_{f}=N_{c}$, we have the same number of $\mu^{u}, \bar{\mu}_{u}$ and $\mu^{1 f}, \bar{\mu}_{f}^{1}$ and the integral over these fermionic moduli yields simply (up to numerical constants which we disregard)

$$
\operatorname{det} \bar{Q} \operatorname{det} \overline{\widetilde{Q}}=\operatorname{det} \bar{M}
$$

where $M_{g}^{f}=\widetilde{Q}_{u}^{f} Q_{g}^{u}$ is the meson superfield matrix. The above expression has to be evaluated at $\bar{\theta}=0$, so that only the scalar components appear.

For $N_{c}=N_{f}=2$ the integral over the bosonic variables has exactly the form considered in Eq. (5.6) of Ref. [54]:

$$
\int d^{3} D d^{4} \bar{w} d^{4} w \mathrm{e}^{-\mathrm{i} D_{c} \bar{w} \tau^{c} w-\bar{w} A w}=\frac{1}{\operatorname{tr} A}
$$

where the $2 \times 2$ matrix $A$ is given by

$$
A_{v}^{u}=\frac{1}{2}\left(Q_{f}^{u} \bar{Q}_{v}^{f}+\overline{\widetilde{Q}}_{f}^{u} \widetilde{Q}_{v}^{f}\right)
$$


Using the D-flatness condition (3.4), it is easy to see that the trace of $A$ can be re-expressed in terms of the low-energy degrees of freedom represented by the meson and baryon superfields $M, B$ and $\tilde{B}$ as follows:

$$
\operatorname{tr} A=(\operatorname{tr} \bar{M} M+\bar{B} B+\overline{\widetilde{B}} \widetilde{B})^{\frac{1}{2}} .
$$

Inserting into Eq. (A.21) the result (A.23) of the fermionic integration and the bosonic integral (A.24) we finally get

$$
W_{\text {n.p. }}=\left.\mathcal{C} \alpha^{\prime 2} \bar{G} \Lambda^{4} \frac{\operatorname{det} \bar{M}}{(\operatorname{tr} \bar{M} M+\bar{B} B+\overline{\widetilde{B}} \widetilde{B})^{1 / 2}}\right|_{\bar{\theta}=0}
$$

as reported in (5.17).

\section{References}

[1] R. Blumenhagen, M. Cvetic, P. Langacker, and G. Shiu, Toward realistic intersecting D-brane models, Ann. Rev. Nucl. Part. Sci. 55 (2005) 71-139, arXiv:hep-th/0502005.

[2] R. Blumenhagen, B. Kors, D. Lust, and S. Stieberger, Four-dimensional String Compactifications with D-Branes, Orientifolds and Fluxes, Phys. Rept. 445 (2007) 1-193, arXiv:hep-th/0610327.

[3] F. Marchesano, Progress in D-brane model building, Fortsch. Phys. 55 (2007) 491-518, arXiv:hep-th/0702094.

[4] E. Cremmer, S. Ferrara, L. Girardello, and A. Van Proeyen, Yang-Mills Theories with Local Supersymmetry: Lagrangian, Transformation Laws and SuperHiggs Effect, Nucl. Phys. B212 (1983) 413.

[5] M. Grana, Flux compactifications in string theory: A comprehensive review, Phys. Rept. 423 (2006) 91-158, arXiv:hep-th/0509003.

[6] M. R. Douglas and S. Kachru, Flux compactification, Rev. Mod. Phys. 79 (2007) 733-796, arXiv:hep-th/0610102.

[7] F. Denef, M. R. Douglas, and S. Kachru, Physics of string flux compactifications, Ann. Rev. Nucl. Part. Sci. 57 (2007) 119-144, arXiv: hep-th/0701050.

[8] S. Gukov, C. Vafa, and E. Witten, CFT's from Calabi-Yau four-folds, Nucl. Phys. B584 (2000) 69-108, arXiv:hep-th/9906070.

[9] T. R. Taylor and C. Vafa, RR flux on Calabi-Yau and partial supersymmetry breaking, Phys. Lett. B474 (2000) 130-137, arXiv:hep-th/9912152.

[10] S. B. Giddings, S. Kachru, and J. Polchinski, Hierarchies from fluxes in string compactifications, Phys. Rev. D66 (2002) 106006, arXiv:hep-th/0105097.

[11] M. Billo, L. Ferro, M. Frau, F. Fucito, A. Lerda, and J. F. Morales, Flux interactions on D-branes and instantons, JHEP 10 (2008) 112, arXiv:0807.1666 [hep-th].

[12] E. Witten, Small Instantons in String Theory, Nucl. Phys. B460 (1996) 541-559, arXiv:hep-th/9511030.

[13] M. R. Douglas, Branes within branes, arXiv:hep-th/9512077. 
[14] F. Denef, M. R. Douglas, B. Florea, A. Grassi, and S. Kachru, Fixing all moduli in a simple F-theory compactification, Adv. Theor. Math. Phys. 9 (2005) 861-929, arXiv:hep-th/0503124.

[15] D. Lust, S. Reffert, W. Schulgin, and S. Stieberger, Moduli stabilization in type IIB orientifolds. I: Orbifold limits, Nucl. Phys. B766 (2007) 68-149, arXiv:hep-th/0506090.

[16] D. Lust, S. Reffert, E. Scheidegger, W. Schulgin, and S. Stieberger, Moduli stabilization in type IIB orientifolds. II, Nucl. Phys. B766 (2007) 178-231, arXiv:hep-th/0609013.

[17] M. B. Green and M. Gutperle, D-instanton induced interactions on a D3-brane, JHEP 02 (2000) 014, arXiv:hep-th/0002011.

[18] M. Billo, M. Frau, I. Pesando, F. Fucito, A. Lerda, and A. Liccardo, Classical gauge instantons from open strings, JHEP 02 (2003) 045, arXiv:hep-th/0211250.

[19] M. Billo, M. Frau, F. Fucito, and A. Lerda, Instanton calculus in $R-R$ background and the topological string, JHEP 11 (2006) 012, arXiv:hep-th/0606013.

[20] N. Akerblom, R. Blumenhagen, D. Lust, E. Plauschinn, and M. Schmidt-Sommerfeld, Non-perturbative SQCD Superpotentials from String Instantons, JHEP 04 (2007) 076, arXiv:hep-th/0612132.

[21] M. Billo, M. Frau, I. Pesando, P. Di Vecchia, A. Lerda, and R. Marotta, Instantons in N=2 magnetized D-brane worlds, JHEP 10 (2007) 091, arXiv:0708.3806 [hep-th].

[22] M. Billo, M. Frau, I. Pesando, P. Di Vecchia, A. Lerda, and R. Marotta, Instanton effects in $N=1$ brane models and the Kahler metric of twisted matter, JHEP 12 (2007) 051, arXiv:0709.0245 [hep-th].

[23] N. Dorey, T. J. Hollowood, V. V. Khoze, and M. P. Mattis, The calculus of many instantons, Phys. Rept. 371 (2002) 231-459, arXiv:hep-th/0206063.

[24] R. Blumenhagen, M. Cvetic, and T. Weigand, Spacetime instanton corrections in $4 D$ string vacua - the seesaw mechanism for D-brane models, Nucl. Phys. B771 (2007) 113-142, arXiv:hep-th/0609191.

[25] L. E. Ibanez and A. M. Uranga, Neutrino Majorana masses from string theory instanton effects, JHEP 03 (2007) 052, arXiv:hep-th/0609213.

[26] B. Florea, S. Kachru, J. McGreevy, and N. Saulina, Stringy instantons and quiver gauge theories, JHEP 05 (2007) 024, arXiv:hep-th/0610003.

[27] M. Bianchi and E. Kiritsis, Non-perturbative and Flux superpotentials for Type I strings on the Z3 orbifold, Nucl. Phys. B782 (2007) 26-50, arXiv:hep-th/0702015.

[28] R. Argurio, M. Bertolini, S. Franco and S. Kachru, Metastable vacua and D-branes at the conifold, JHEP 06 (2007) 017, arXiv:hep-th/0703263.

[29] R. Argurio, M. Bertolini, G. Ferretti, A. Lerda, and C. Petersson, Stringy Instantons at Orbifold Singularities, JHEP 06 (2007) 067, arXiv:0704.0262 [hep-th].

[30] M. Bianchi, F. Fucito, and J. F. Morales, D-brane Instantons on the $T^{6} / Z_{3}$ orientifold, JHEP 07 (2007) 038, arXiv:0704.0784 [hep-th].

[31] L. E. Ibanez, A. N. Schellekens, and A. M. Uranga, Instanton Induced Neutrino Majorana Masses in CFT Orientifolds with MSSM-like spectra, JHEP 06 (2007) 011, arXiv:0704.1079 [hep-th]. 
[32] S. Antusch, L. E. Ibanez, and T. Macri, Neutrino Masses and Mixings from String Theory Instantons, JHEP 09 (2007) 087, arXiv:0706.2132 [hep-ph].

[33] R. Blumenhagen, M. Cvetic, D. Lust, R. Richter, and T. Weigand, Non-perturbative Yukawa Couplings from String Instantons, Phys. Rev. Lett. 100 (2008) 061602, arXiv:0707.1871 [hep-th].

[34] O. Aharony and S. Kachru, Stringy Instantons and Cascading Quivers, JHEP 09 (2007) 060, arXiv:0707.3126 [hep-th].

[35] R. Blumenhagen, M. Cvetic, R. Richter, and T. Weigand, Lifting D-Instanton Zero Modes by Recombination and Background Fluxes, JHEP 10 (2007) 098, arXiv:0708.0403 [hep-th].

[36] P. G. Camara, E. Dudas, T. Maillard, and G. Pradisi, String instantons, fluxes and moduli stabilization, Nucl. Phys. B795 (2008) 453-489, arXiv:0710.3080 [hep-th].

[37] L. E. Ibanez and A. M. Uranga, Instanton Induced Open String Superpotentials and Branes at Singularities, JHEP 02 (2008) 103, arXiv:0711.1316 [hep-th].

[38] I. Garcia-Etxebarria and A. M. Uranga, Non-perturbative superpotentials across lines of marginal stability, JHEP 01 (2008) 033, arXiv:0711.1430 [hep-th].

[39] C. Petersson, Superpotentials From Stringy Instantons Without Orientifolds, JHEP 05 (2008) 078, arXiv:0711.1837 [hep-th].

[40] M. Bianchi and J. F. Morales, Unoriented D-brane Instantons vs Heterotic worldsheet Instantons, JHEP 02 (2008) 073, arXiv:0712.1895 [hep-th].

[41] R. Blumenhagen and M. Schmidt-Sommerfeld, Power Towers of String Instantons for $N=1$ Vacua, arXiv:0803.1562 [hep-th].

[42] R. Argurio, G. Ferretti, and C. Petersson, Instantons and Toric Quiver Gauge Theories, arXiv:0803.2041 [hep-th].

[43] M. Cvetic, R. Richter, and T. Weigand, (Non-)BPS bound states and D-brane instantons, arXiv:0803.2513 [hep-th].

[44] S. Kachru and D. Simic, Stringy Instantons in IIB Brane Systems, arXiv:0803.2514 [hep-th].

[45] I. Garcia-Etxebarria, F. Marchesano, and A. M. Uranga, Non-perturbative F-terms across lines of BPS stability, arXiv:0805.0713 [hep-th].

[46] M. Buican and S. Franco, SUSY breaking mediation by D-brane instantons, arXiv:0806.1964 [hep-th].

[47] P. G. Camara, L. E. Ibanez, and A. M. Uranga, Flux-induced SUSY-breaking soft terms, Nucl. Phys. B689 (2004) 195-242, arXiv:hep-th/0311241.

[48] P. G. Camara, L. E. Ibanez, and A. M. Uranga, Flux-induced SUSY-breaking soft terms on D7-D3 brane systems, Nucl. Phys. B708 (2005) 268-316, arXiv:hep-th/0408036.

[49] I. Affleck, M. Dine, and N. Seiberg, Dynamical Supersymmetry Breaking in Supersymmetric QCD, Nucl. Phys. B241 (1984) 493-534.

[50] M. Bertolini, P. Di Vecchia, G. Ferretti, and R. Marotta, Fractional branes and $N=1$ gauge theories, Nucl. Phys. B630 (2002) 222-240, arXiv:hep-th/0112187. 
[51] K. Intriligator and N. Seiberg, The runaway quiver, JHEP 02 (2006) 031, arXiv:hep-th/0512347.

[52] S. Franco, A. Hanany, F. Saad, and A. M. Uranga, Fractional branes and dynamical supersymmetry breaking, JHEP 01 (2006) 011, arXiv:hep-th/0505040.

[53] K. A. Intriligator and N. Seiberg, Lectures on supersymmetric gauge theories and electricmagnetic duality, Nucl. Phys. Proc. Suppl. 45BC (1996) 1-28, arXiv:hep-th/9509066.

[54] Y. Matsuo, J. Park, C. Ryou, and M. Yamamoto, D-instanton derivation of multi-fermion F-terms in supersymmetric QCD, JHEP 06 (2008) 051, arXiv:0803.0798 [hep-th].

[55] C. Beasley and E. Witten, New instanton effects in supersymmetric QCD, JHEP 01 (2005) 056, arXiv:hep-th/0409149.

[56] M. Billo, M. Frau, F. Lonegro and A. Lerda, $N=1 / 2$ quiver gauge theories from open strings with $R$-R fluxes, JHEP 05 (2005) 047, arXiv: hep-th/0502084.

[57] A. M. Uranga, D-brane instantons and the effective field theory of flux compactifications, arXiv:0808.2918 [hep-th]. 